Reactor and Nuclear Systems Division

\title{
Proceedings of the \\ Nuclear Data Roadmapping and Enhancement Workshop (NDREW) for Nonproliferation
}

Catherine Romano ${ }^{1}$, Timothy Ault ${ }^{2}$, Lee Bernstein ${ }^{3}$, Rian Bahran ${ }^{4}$, Bradley Rearden ${ }^{1}$, Patrick Talou ${ }^{4}$, Brian Quiter ${ }^{3}$, Sara Pozzi ${ }^{5}$, Matt Devlin ${ }^{4}$, Jason Burke ${ }^{6}$, Todd Bredeweg ${ }^{4}$, Elizabeth McCutchan ${ }^{7}$, Sean Stave $^{8}$, Teresa Bailey ${ }^{6}$, Susan Hogle ${ }^{1}$, Christopher Chapman ${ }^{1}$, Aaron Hurst ${ }^{3}$, Noel Nelson ${ }^{2}$, Fredrik Tovesson ${ }^{2}$, Donald Hornback ${ }^{2}$

${ }^{1}$ Oak Ridge National Laboratory

${ }^{2}$ National Nuclear Security Administration / NA-22

${ }^{3}$ Lawrence Berkeley National Laboratory

${ }^{4}$ Los Alamos National Laboratory

${ }^{5}$ University of Michigan

${ }^{6}$ Lawrence Livermore National Laboratory

${ }^{7}$ Brookhaven National Laboratory

${ }^{8}$ National Nuclear Security Administration / NA-24

Date Published: April 24, 2018

Prepared by

OAK RIDGE NATIONAL LABORATORY

Oak Ridge, TN 37831-6283

managed by

UT-BATTELLE, LLC

for the

US DEPARTMENT OF ENERGY

under contract DE-AC05-00OR22725 


\title{
DOCUMENT AVAILABILITY
}

Reports produced after January 1, 1996, are generally available free via US Department of Energy (DOE) SciTech Connect.

\section{Website www.osti.gov}

Reports produced before January 1, 1996, may be purchased by members of the public from the following source:

\author{
National Technical Information Service \\ 5285 Port Royal Road \\ Springfield, VA 22161 \\ Telephone 703-605-6000 (1-800-553-6847) \\ TDD 703-487-4639 \\ Fax 703-605-6900 \\ E-mail info@ntis.gov \\ Website http://classic.ntis.gov/
}

Reports are available to DOE employees, DOE contractors, Energy Technology Data Exchange representatives, and International Nuclear Information System representatives from the following source:

Office of Scientific and Technical Information

PO Box 62

Oak Ridge, TN 37831

Telephone 865-576-8401

Fax 865-576-5728

E-mail reports@osti.gov

Website http://www.osti.gov/contact.html

This report was prepared as an account of work sponsored by an agency of the United States Government. Neither the United States Government nor any agency thereof, nor any of their employees, makes any warranty, express or implied, or assumes any legal liability or responsibility for the accuracy, completeness, or usefulness of any information, apparatus, product, or process disclosed, or represents that its use would not infringe privately owned rights. Reference herein to any specific commercial product, process, or service by trade name, trademark, manufacturer, or otherwise, does not necessarily constitute or imply its endorsement, recommendation, or favoring by the United States Government or any agency thereof. The views and opinions of authors expressed herein do not necessarily state or reflect those of the United States Government or any agency thereof. 


\section{TABLE OF CONTENTS}

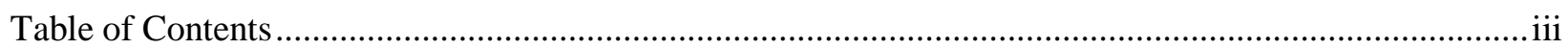

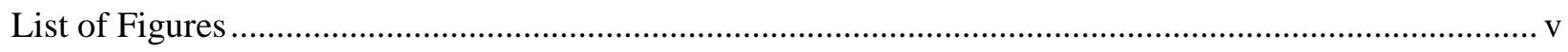

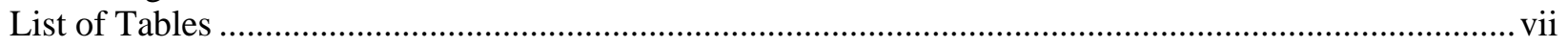

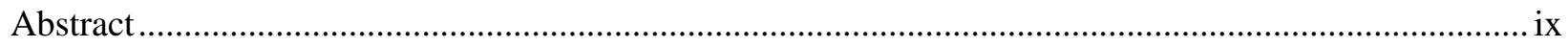

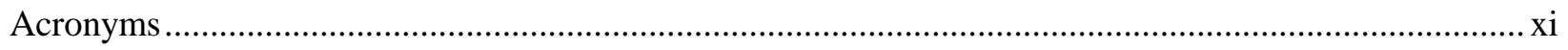

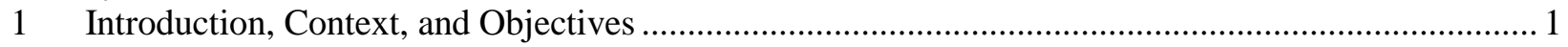

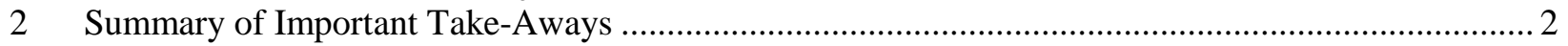

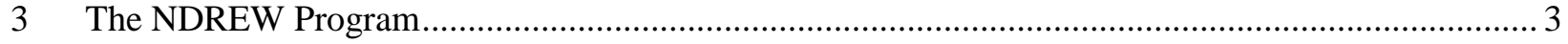

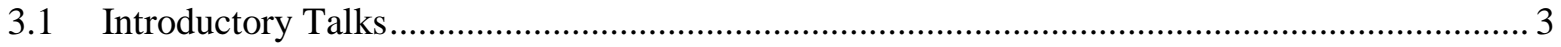

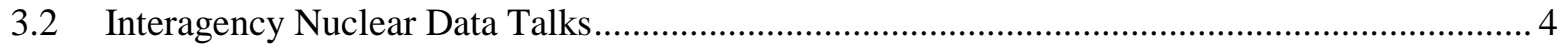

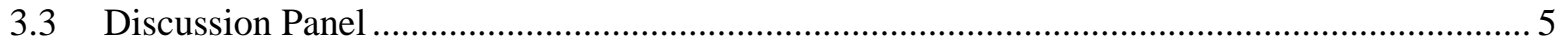

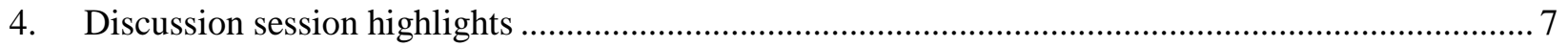

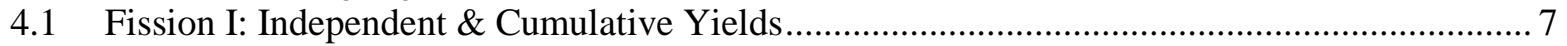

4.2 Fission II: Prompt Neutron and Gamma Ray Emissions .................................................... 7

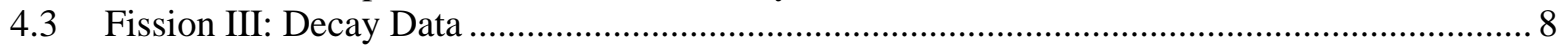

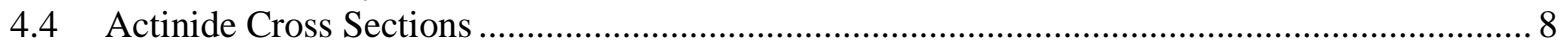

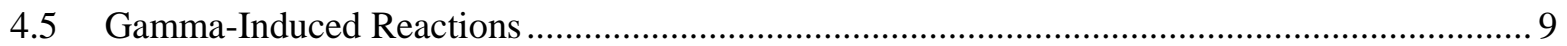

4.6 Neutron Capture and Associated Spectra and Inelastic Scattering and Associated Spectra ....... 9

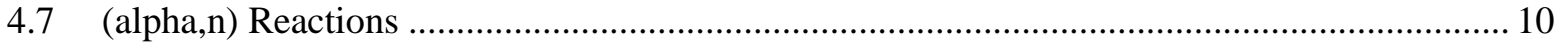

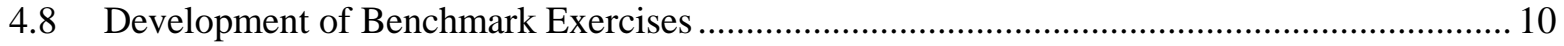

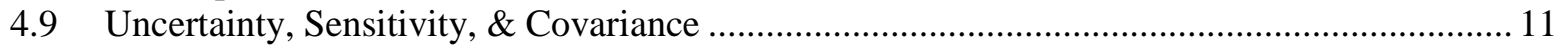

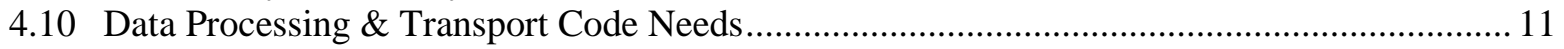

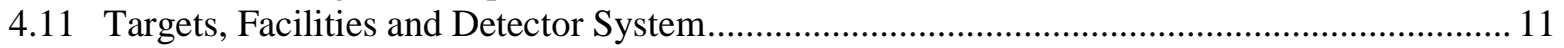

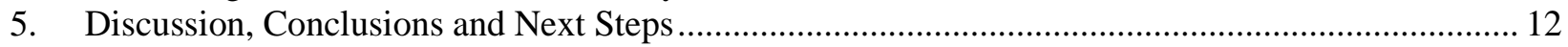

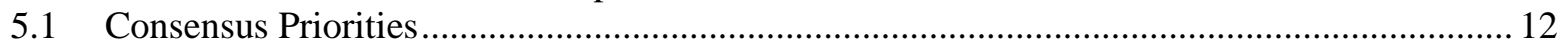

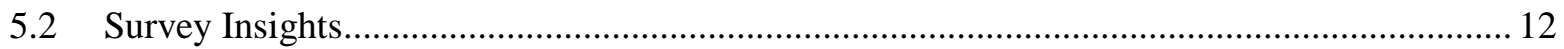

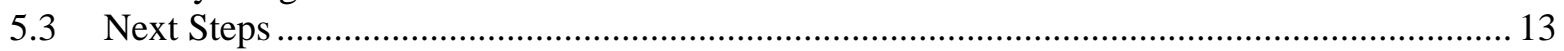

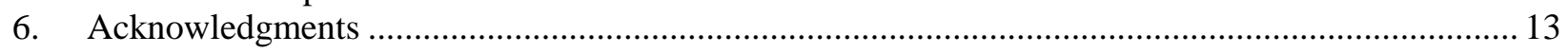

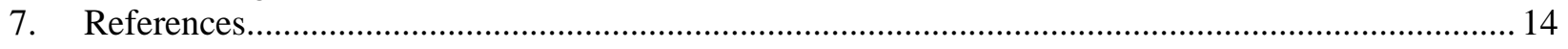

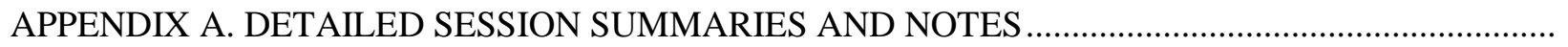

A.1 Fission I: Independent and Cumulative Fission Yields.................................................. A-1

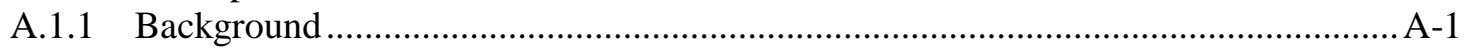

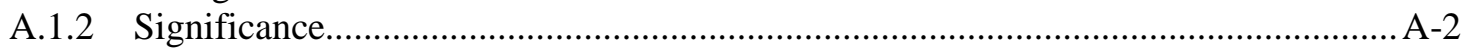

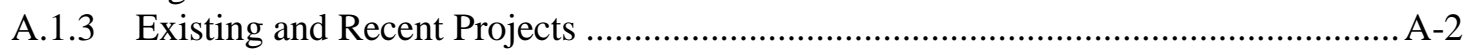

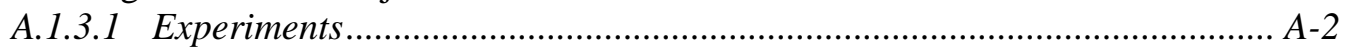

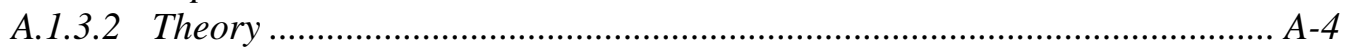

A.1.3.3 Models \& Evaluations.................................................................... A-4

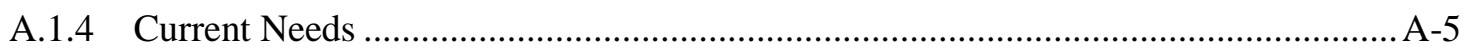

A.1.4.1 Experimental Needs................................................................... A-5

A.1.4.2 Theory Needs.................................................................................... A-5

A.1.4.3 Modeling / Computation Needs ............................................................... A-5

A.1.4.4 Evaluation and Data Format Needs..................................................... A-6

A.1.4.5 Workforce/Infrastructure Needs........................................................ A-6

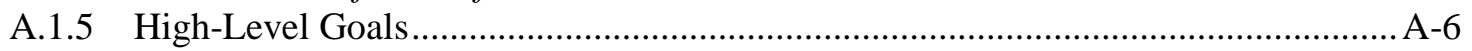

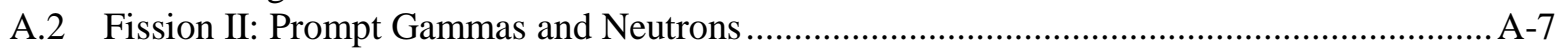

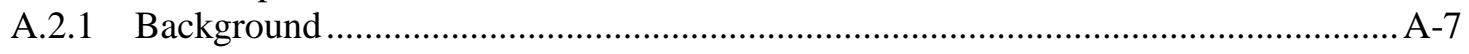

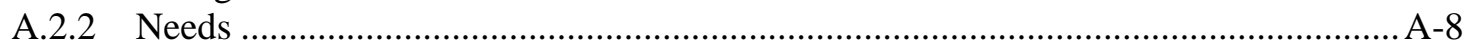

A.2.3 Physics and Transport Codes ............................................................................ A-9 


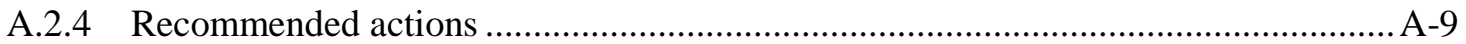

A.2.4.1 Facilities in the United States .............................................................. A-9

A.2.4.2 International Facilities........................................................................ A-10

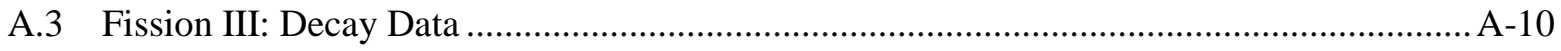

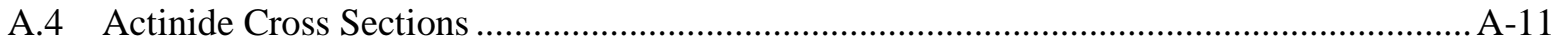

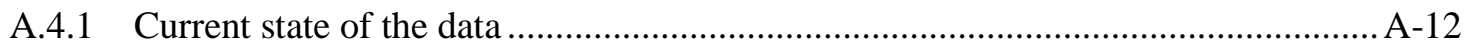

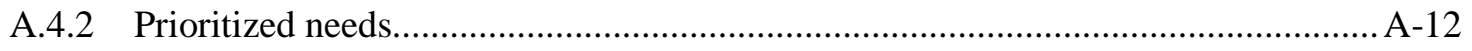

A.4.2.1 Isotopes of Interest ........................................................................... A-12

A.4.2.2 Structure-Informed Reaction Modeling Needs...................................... A-13

A.4.2.3 Facility and Target Needs ..................................................................... A-13

A.4.2.4 Evaluation and Uncertainty Needs...................................................... A-13

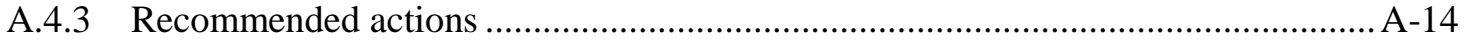

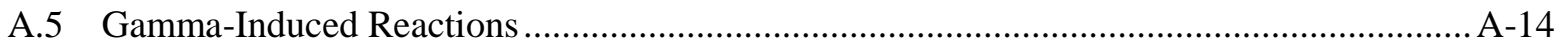

A.5.1 Data assessment for photofission model-simulation comparison .......................... A-15

A.5.2 Other photonuclear data applications............................................................... A-15

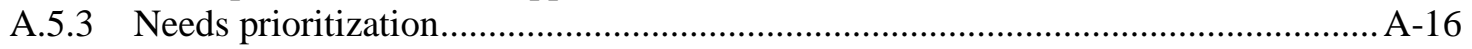

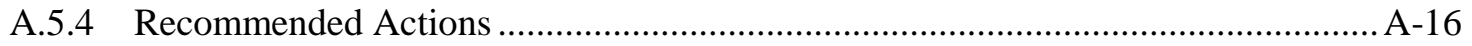

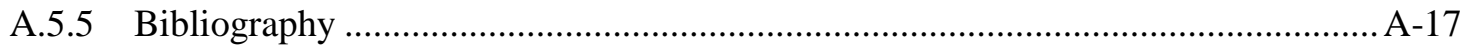

A.6 Neutron Capture and Associated Spectra, and Inelastic Neutron Scattering and Associated Spectra (combined summary) .....................................................................

A.6.1 Prioritized Plan of Action - Capture Gammas.....................................................A-19

A.6.2 Prioritized Plan of Action - Inelastic Scattering ................................................ A-19

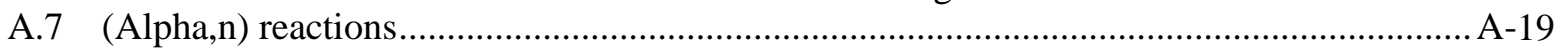

A.8 Development of Benchmark Exercises ................................................................... A-20

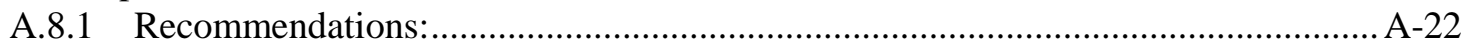

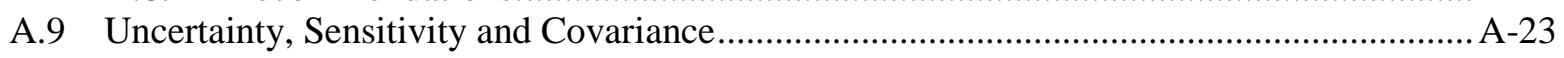

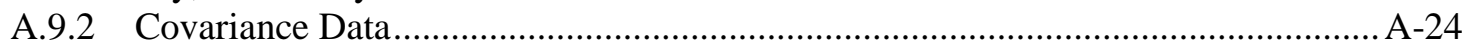

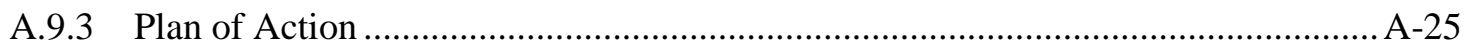

A.10 Data Processing and Transport Code Needs ................................................................. A-26

A.10.1 Nuclear Data Processing Codes ................................................................... A-27

A.10.1.1 Transport code library processing .................................................. A-27

A.10.1.2 Transmutation data processing .......................................................... A-27

A.10.1.3 Nuclear data uncertainty processing .................................................. A-28

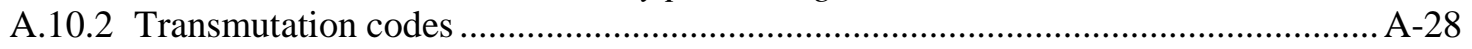

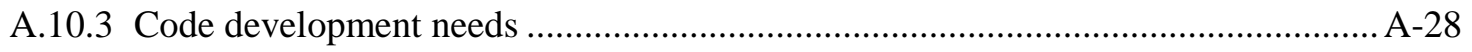

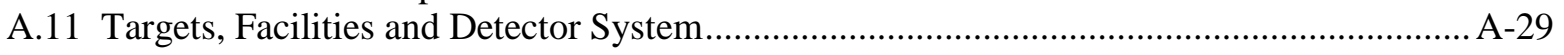

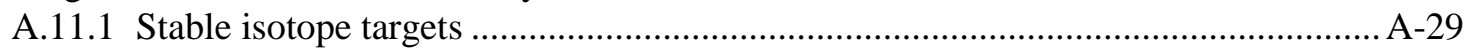

A.11.1.1 Enriched stable isotope availability ................................................... A-29

A.11.1.2 Target fabrication of stable isotopes.................................................. A-29

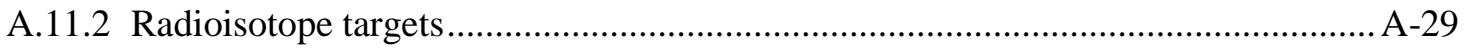

A.11.2.1 Enriched radioisotope availability ....................................................... A-29

A.11.2.2 Target fabrication of radioisotopes................................................... A-30

A.11.2.3 Target characterization ................................................................. A-30

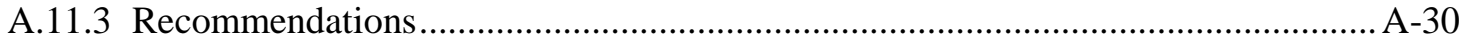

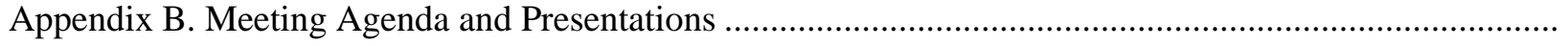

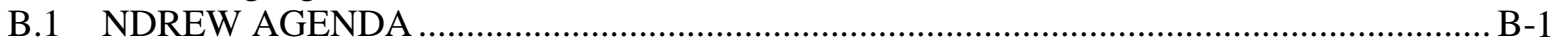

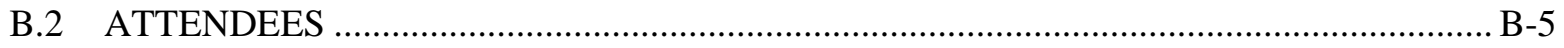




\section{LIST OF FIGURES}

Figure 1. NDREW participants, January 2018. .................................................................................. 3

Figure 2. Illustration of the process to measure, evaluate and test nuclear data and the propagation of uncertainties.....

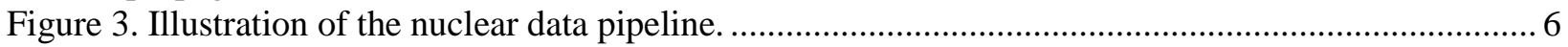

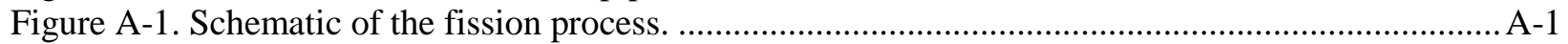

Figure A-2. The actinide network of capture and decay ................................................................... A-12

Figure A-3. Flow of information to validate nuclear data libraries using integral experiments............. A-21

Figure A-4. Decay heat uncertainty as a function of burnup and cooling time. ................................... A-22 



\section{LIST OF TABLES}

Table A.10-1. ENDF Reaction Cross Section and Fission Multiplicity Processing Tools.................... A-27 



\begin{abstract}
On January 23-25, 2018 in Washington, DC, the National Nuclear Security Administration (NNSA) Office of Defense Nuclear Nonproliferation (DNN) Research and Development (NA-22) supported the three-day Nuclear Data Roadmapping and Enhancement Workshop (NDREW) to enable nonproliferation missions. The goal was to assemble users and producers of nuclear data to provide input to identify nuclear data needs, suggest solutions to address these needs, and rank potential solutions with regard to mission impact. The workshop was organized into 12 discussion sessions which were bookended by introductory and concluding presentations. Eight discussion topics were defined by specific nuclear reaction or data types, while the other four covered infrastructure, benchmarks, uncertainties, and code development needs. Over 110 attendees represented national laboratories, universities, and headquarters, as well as international collaborators and industry representatives. Based on survey results and other feedback, attendees found the workshop valuable since it initiated a new conversation between programs and provided collaborative opportunities across stakeholder groups such as data users and experimentalists/evaluators. The proceedings presented herein summarize the content of the workshop, provide session notes and important highlights, and documents recommendations from the attendees.
\end{abstract}




\section{ACRONYMS}

\begin{tabular}{|c|c|}
\hline ACE & A Compact ENDF \\
\hline AFTAC & Air Force Technical Applications Center \\
\hline AMPX & A Modern Cross Section Processing System \\
\hline ANL & Argonne National Laboratory \\
\hline API & application programming interface \\
\hline ASCI & American Standard Code for Information Interchange \\
\hline ASCR & Advanced Scientific Computing Research \\
\hline BNL & Brookhaven National Laboratory \\
\hline CARIBU & Californium Rare Isotope Breeder Upgrade \\
\hline $\mathrm{CE}$ & continuous energy \\
\hline CEA & French Atomic Energy and Alternative Energies Commission \\
\hline CERN-NTOF & European Organization for Nuclear Research - Neutron Time-of-Flight \\
\hline CFY & cumulative fission yields \\
\hline CGM & Cascading Gamma-Ray Multiplicity \\
\hline CGMF & Upgrade to CGM for fission \\
\hline CIELO & Collaborative International Evaluated Library Organization \\
\hline CINDER & Code System for Actinide Transmutation Calculations \\
\hline COG & multi-particle transport code \\
\hline CRP & coordinated research project \\
\hline CSEWG & Cross Section Evaluation Working Group \\
\hline CWMDT & Countering Weapons of Mass Destruction \\
\hline DAF & Device Assembly Facility \\
\hline DANCE & Detector for Advanced Neutron Capture Experiments \\
\hline DD & fusion of deuterium atoms \\
\hline DHS & US Department of Homeland Security \\
\hline DNDO & Domestic Nuclear Detection Office \\
\hline DNN & Office of Defence Nuclear Nonproliferation \\
\hline DOE & US Department of Energy \\
\hline DT & fusion of a deuterium and a tritium atom \\
\hline DTRA & Defense Threat Reduction Agency \\
\hline EGAF & evaluated gamma activation file \\
\hline ENDF & evaluated nuclear data file \\
\hline ENDL & evaluated nuclear data library \\
\hline ENSDF & evaluated nuclear structure data file \\
\hline EPA & Environmental Protection Agency \\
\hline EXFOR & experimental nuclear reaction data \\
\hline FOA & funding opportunity announcement \\
\hline FREYA & Fission Reaction Event Yield Algorithm \\
\hline FRIB & Facility for the Production of Rare Isotope Beams \\
\hline FUDGE & A Toolkit for Nuclear Data Management and Processing \\
\hline GAINS & Gamma Array for Neutron Inelastic Scattering \\
\hline GADRAS & Gamma Detector Response and Analysis Software \\
\hline GDR & giant dipole resonance \\
\hline GEANT & Geometry and Tracking \\
\hline GEF & General Fission \\
\hline GELINA & Geel Linear Accelerator \\
\hline GNDS & General Nuclear Data Structure \\
\hline HIGS & High Intensity Gamma-Ray Source \\
\hline HKED & Hybrid K-Edge Densitometer Laboratory \\
\hline IAEA & International Atomic Energy Agency \\
\hline ICRP & International Commission on Radiation Protection \\
\hline IFY & independent fission yields \\
\hline INL & Idaho National Laboratory \\
\hline JEFF & Joint Evaluated Fission and Fusion \\
\hline
\end{tabular}




\begin{tabular}{|c|c|}
\hline JNST & Journal of Nuclear Science and Technology \\
\hline LANL & Los Alamos National Laboratory \\
\hline LANSCE & Los Alamos Neutron Science Center \\
\hline LBNL & Lawrence Berkeley National Laboratory \\
\hline LINAC & Linear Accelerator \\
\hline LLNL & Lawrence Livermore National Laboratory \\
\hline MCNP & Monte Carlo N-Particle \\
\hline MG & multigroup \\
\hline MIT & Massachusetts Institute of Technology \\
\hline MTAS & Modular Total Absorption Spectrometer \\
\hline MSU & Michigan State University \\
\hline NA-22 & DNN R\&D \\
\hline NA-24 & DNN Office of Nonproliferation and International Security \\
\hline NA-221 & DNN Office of Proliferation Detection \\
\hline NA-241 & DNN Nuclear Safeguards and Security \\
\hline NCERC & National Criticality Experiments Research Center \\
\hline NCSP & Nuclear Criticality Safety Program \\
\hline NCSU & North Carolina State University \\
\hline NDA & nondestructive assay \\
\hline NDEM & Nuclear Data Exchange Meeting \\
\hline NDIAWG & Nuclear Data Interagency Working Group \\
\hline NDNCA & Nuclear Data Needs and Capabilities for Applications \\
\hline NDREW & Nuclear Data Roadmapping and Enhancement Workshop \\
\hline NDWG & Nuclear Data Working Group \\
\hline NE & Office of Nuclear Energy \\
\hline NEA & Nuclear Energy Agency \\
\hline NEUANCE & neutron detector array \\
\hline NEWT & New Transport Algorithm code in SCALE \\
\hline NRF & nuclear resonance fluorescence \\
\hline NIST & National Institute of Standards and Technology \\
\hline NJOY & nuclear data processing code \\
\hline NNDC & National Nuclear Data Center \\
\hline NNSA & National Nuclear Security Administration \\
\hline NP & Office of Nuclear Physics \\
\hline NRF & nuclear resonance fluorescence \\
\hline OECD & Organisation for Economic Cooperation and Development \\
\hline ORNL & Oak Ridge National Laboratory \\
\hline ORIGEN & Oak Ridge Isotope Generation \\
\hline ORSEN & ORIGEN Sensitivity Analysis \\
\hline PANDA & passive nondestructive assay (manual) \\
\hline PFNS & prompt fission neutron spectra \\
\hline PI & principal investigator \\
\hline PNNL & Pacific Northwest National Laboratory \\
\hline $\mathrm{R} \& \mathrm{D}$ & research and development \\
\hline RPI & Rensselaer Polytechnic Institute \\
\hline RTFDB & Research and Test Facilities Database \\
\hline SCALE & Standardized Computer Analyses for Licensing Evaluation \\
\hline SLAC & Stanford Linear Accelerator Center \\
\hline SNL & Sandia National Laboratory \\
\hline TAGS & total absorption gamma-ray spectroscopy \\
\hline TALYS & software for the simulation of nuclear reactions \\
\hline TDHF & time-dependent constrained Hartree-Fock \\
\hline TD-SLDA & Time. Dependent Superfluid Local Density Approximation \\
\hline TENDL & TALYS-based Evaluated Nuclear Data Library \\
\hline TKE & total kinetic energy \\
\hline TOF & time of flight \\
\hline
\end{tabular}


TSUNAMI

TUNL

UCB

Tools for Sensitivity and Uncertainty Analysis Methodology Implementation

$\mathrm{UM}$ Triangle Universities Nuclear Laboratory University of California, Berkeley

USNDP

$\mathrm{XRF}$

University of Michigan

US Nuclear Data Program

$\mathrm{x}$-ray fluorescence

XSDRNPM

discrete ordinates code which solves the one-dimensional Boltzmann equation 



\section{INTRODUCTION, CONTEXT, AND OBJECTIVES}

The National Nuclear Security Administration (NNSA) Office of Defense Nuclear Nonproliferation Research and Development (DNN R\&D or NA-22) oversees a spectrum of efforts to "detect, identify, and characterize: (1) foreign nuclear weapons programs, (2) illicit diversion of special nuclear materials, and (3) global nuclear detonations" [1]. These efforts entail the application, development, and adaptation of a variety of techniques, methods, models, and technologies which require a comprehensive understanding of pertinent nuclear reactions and properties. The key parameters associated with these reactions and properties-including cross sections, branching ratios, half-lives, gamma decay energies, neutron-gamma correlations, and many others frequently have major impacts on the overall uncertainty of a measurement or simulation, and in some cases, nuclear data serve as the dominant contributor to uncertainty. Therefore, NA-22 has a vested interest in improving nuclear data in a manner that augments the capabilities of end users.

The National Nuclear Data Center (NNDC), based at Brookhaven National Laboratory, compiles, evaluates, formats, and maintains nuclear data as part of the Evaluated Nuclear Data Files (ENDF) [2]; similar counterparts exist around the world. However, the burden of ensuring that nuclear data are measured, evaluated, and processed into modeling and simulation tools falls primarily on organizations who use the data. Since 2007, DNN R\&D has invested in nuclear data improvements, spending more than \$25 million on projects focused on nuclear data. To date, these expenditures have largely been narrowly focused to serve the specific needs of individual mission areas, and there has been a growing recognition that a more strategic approach is needed. Nuclear data needs are shared across the various nonproliferation missions, although each mission often places emphasis on different aspects and applications of the data. Furthermore, many nuclear data are cross cutting, impacting nuclear energy, isotope production, and basic science.

Based on these data needs, DNN R\&D and collaborating national laboratories set out to organize the Nuclear Data Roadmapping and Enhancement Workshop (NDREW). This workshop was intended to obtain input from nuclear data users and producers to create a strategic document to guide nuclear data funding for the next five to ten years for nonproliferation missions supported by DNN R\&D. Based on mission and technology development priorities, the nuclear data strategy will provide recommendations, document the current state of the data, and determine the expected impacts of reduced uncertainties in nuclear data.

NDREW built on lessons from several recent collaborations, the foremost being the "Nuclear Data Needs and Capabilities for Applications" (NDNCA) workshop hosted by the DOE Office of Nuclear Physics (DOE NP) and DNN R\&D at Lawrence Berkeley National Laboratory in 2015 [3]. The workshop targeted needs for all applications and achieved its goal of collecting expert opinions from users on a broad range of nuclear data needs. This created a strong foundation for future discussions. The published list of nuclear data needs includes several cross-cutting areas in which multiple users can benefit from data improvements. As a result of NDNCA, the Nuclear Data Working Group (NDWG) was formed to facilitate cross-program communication and was made up of representatives designated by program offices with an interest in nuclear data collaboration [4]. The NDWG identified and prioritized several of the most important cross cutting nuclear data needs and presented a proposed solution, as well as general recommendations for funding nuclear data, to 25 federal program representatives at the Nuclear Data Exchange Meeting (NDEM) on April 15, 2016 in Washington, DC. The NDEM provided an opportunity for critical conversations between the nuclear data community and program managers to provide guidance in resolving nuclear data needs. 
After the NDEM, the program managers created a Nuclear Data Interagency Working Group (NDIAWG) that was chaired by DOE NP to coordinate nuclear data funding between participating program offices, including DNN R\&D. The result was the 2017 NDIAWG Funding Opportunity Announcement (FOA), which was managed by DOE NP for all programs [5]. Participants in the FOA included DOE NP, the Office of Nuclear Energy (NE), and the Isotope Program (IP) of the Office of Science (SC), DNN R\&D, and the Domestic Nuclear Detection Office (DNDO) of the Department of Homeland Security (DHS). The 2017 FOA resulted in funding for three projects:

1. Improving the Nuclear Data on Fission Product Decays at CARIBU PI: Savard, Guy (ANL)

2. Novel Approach for Improving Antineutrino Spectral Predictions for Nonproliferation Applications PI: Kondev, Filip (ANL)

3. ${ }^{238} U(p, x n)$ and ${ }^{235} U(d, x n) ~{ }^{235-237} N p$ Nuclear Reaction Cross Sections Relevant to the Production of ${ }^{236 \mathrm{~g}} \mathrm{~Np}$

PI: Fassbender, Michael (LANL)

In preparation for DNN R\&D participation in future NDIAWG FOAs, NDREW was configured to achieve the following four objectives:

1. To collect subject matter expert input, including nuclear data prioritization and recommended solutions.

2. To ensure that the resulting nonproliferation strategic document captures the appropriate intersections among ongoing efforts of other programs.

3. To facilitate communication and collaboration among programs and organizations dependent on nuclear data.

4. To increase mutual awareness and understanding of different stakeholder segments of the nuclear data community, including experimentalists, evaluators, end users, and program managers.

\section{SUMMARY OF IMPORTANT TAKE-AWAYS}

Several overarching workshop takeaways that do not fit into a specific topic area are listed below.

1. Representation at Nuclear Data Week, the annual meeting of the USNDP and the Cross Section Evaluation Working Group (CSEWG), will be critical for ensuring that each programs' needs are considered and addressed as new ENDF files are developed.

2. Workforce development and facility maintenance are important for ensuring long-term improvements in nuclear data. Ongoing efforts will require collaboration among multiple laboratories and universities, maximizing the strengths of each.

3. New measurement and evaluation efforts must be well integrated into the USNDP.

4. All nuclear data efforts' end goal should be the incorporation of that data into the databases with the goal of reaching the end users.

5. Continued communication, collaboration and cooperation between mission programs with input from the nuclear data community will be key to ensuring that nuclear data funding provides the highest level of mission impact while improving efficiency. The NDIAWG FOA has demonstrated its utility for this purpose.

6. Continued communication between nuclear data users and producers is important to determine nuclear data priorities and to keep the nuclear data producers focused on 
mission needs. There is consensus from NDREW participants that this communication is useful.

7. New nuclear data projects should address the modeling and data processing required to use the new data. As nuclear data and covariances become more complex, the processing codes will require enhanced capabilities to handle the large files.

8. NDREW participants concurred that they found the workshop valuable since it initiated conversation and presented new opportunities for collaboration among data users, nuclear data experimentalists, and evaluators.

\section{THE NDREW PROGRAM}

NDREW was held January 23-25, 2018, in Washington, DC. It was organized into 12 discussion sessions, along with introductory and concluding presentations. Over 110 attendees represented national laboratories, universities, and headquarters, as well as international collaborators and industry representatives. The agenda and attendees are listed in Appendix B, and a group photo is provided in Figure 1. The consensus was that attendees found the workshop valuable since it initiated conversation and presented new opportunities for collaboration among data users, nuclear data experimentalists, and nuclear data evaluators.

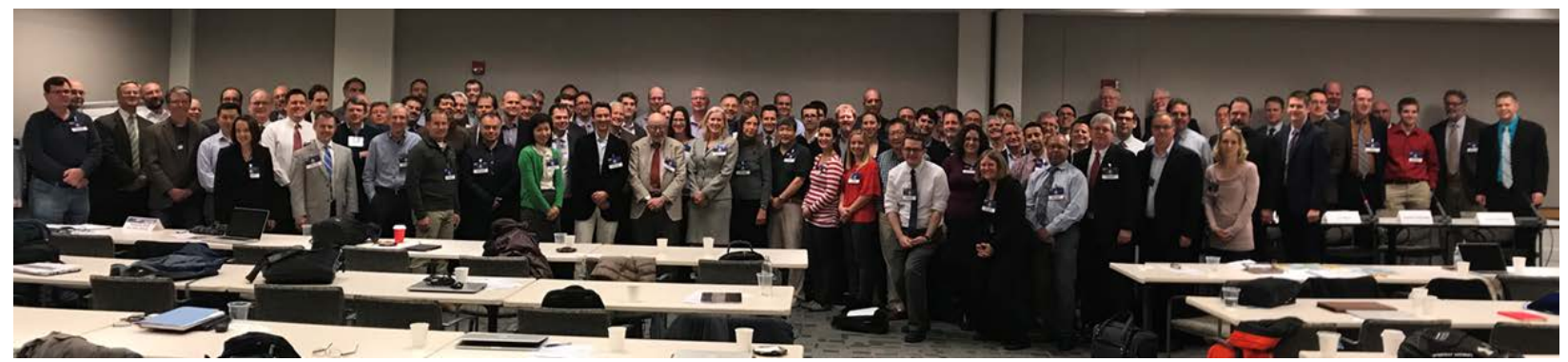

Figure 1. NDREW participants, January 2018.

\subsection{INTRODUCTORY TALKS}

The first morning of the workshop included presentations organized into three parts: introductory talks from interagency participants on nuclear data needs, program managers from other nonproliferation organizations sharing their connections to nuclear data issues, and a presentation/panel session emphasizing the perspectives data end users and curators.

Craig Sloan, Director of Proliferation Detection (NA-221) within DNN R\&D, presented the welcome address. He described several nuclear data projects recently funded by DNN R\&D, including Radiochemistry for americium cross section measurements, development of CGMF [6] and Fission Reaction Event Yield Algorithm (FREYA) [7], computational tools integrated into MCNP [8] transport code for processing correlated neutron and gamma emission from fission, surrogate cross section measurements, and ${ }^{19} \mathrm{~F}(\alpha, \mathrm{n})$ cross sections measurements.

Catherine Romano of Oak Ridge National Laboratory discussed lessons from the prior NDNCA and NDEM gatherings and reiterated the goals of the workshop, previewing the content for upcoming days. She tasked participants to work collaboratively and focus on the nuclear data solutions with the most mission impact. 


\subsection{INTERAGENCY NUCLEAR DATA TALKS}

Tim Hallman, Associate Director of Nuclear Physics, described his office's support of the USNDP and their goal of collaborating with other offices in support of application-driven nuclear data. The collaboration is being accomplished through the NDIAWG and started with last year's first annual nuclear data FOA. The 2018 NDIAWG FOA was released at the time of this writing [9]. Participants include DOE NP and Advanced Scientific Computing Research (ASCR) programs within the Office of Science (SC), the Office of Nuclear Energy (NE), and the Offices of DNN R\&D and Experimental Science (NA-113) of NNSA. The focus of the announcement is solicitation for improvements to the nuclear data pipeline to enable incorporation of nuclear data into the databases more readily, application of advanced computing techniques to nuclear data, nuclear data measurements for advanced reactors, and new fission yield evaluations.

Craig Sloan presented for Bill Junek, Senior Scientist for the Air Force Technical Applications Center (AFTAC) whose mission is to monitor international compliance with nuclear test ban treaties. AFTAC's research and development nuclear data needs are covered in their Research and Development Roadmap. These needs include fission cross sections and fission yields of actinides induced by neutrons in the midenergy $(0.1 \mathrm{keV}-1 \mathrm{MeV})$ range. Actinides of interest include those other than ${ }^{235} \mathrm{U}$ or ${ }^{239} \mathrm{Pu}$. Decay data are also important for isotopes such as ${ }^{153} \mathrm{Sm}$ and ${ }^{115 \mathrm{~m}} \mathrm{Cd}$.

Kevin Mueller, nuclear data lead for the Defense Threat Reduction Agency (DTRA), described the DTRA mission space in nuclear detection, post-detonation forensics, weapons effects, and nuclear survivability. DTRA is working to establish their needs, and they are finding that improved communication between nuclear data users and producers would be helpful. Efforts of interest include:

- $\quad$ Evaluations of (n, $\gamma$ ), (n, n'), (n, 2n), (n, 3n), and (n, f) reactions for ${ }^{236} \mathrm{Pu},{ }^{237} \mathrm{Pu}$, and ${ }^{238} \mathrm{Pu}$ using new fission cross section data with TALYS code [10].

- $\quad{ }^{191,193} \operatorname{Ir}(n, 2 n)^{190,192} \operatorname{Ir}$ measurements

- $\quad{ }^{63,65} \mathrm{Cu}(\mathrm{n}, \gamma){ }^{64,66} \mathrm{Cu}$ measurements

- Short lived (<5 minutes) neutron-induced fission product yield measurements with plans to explore fission products existing $<1$ second.

- $\quad \mathrm{Co}, \mathrm{Cu}$, Ta and Fe high energy (0.5 - $20 \mathrm{MeV})$ transmission and (n, $\gamma$ ) measurements (RPI)

- Investigation of fission product yields from thermal to fast energy fission induced by both neutrons and photons

- The time-dependent neutron/gamma intensities resulting from short-lived fission fragments produced by fast neutron fission

Tim Ashenfelter, Program Manager, Transformational and Applied Research Directorate of DNDO described the agency's mission to address forensics and nuclear materials detection with a focus on security at borders and ports. DNDO is currently employing active gamma interrogation up to $9 \mathrm{MeV}$, requiring improved fission cross sections and correlated neutron emission information for 6-9 $\mathrm{MeV}$ incident gammas.

Arjan Koning, Head of the Nuclear Data Section of the International Atomic Energy Agency (IAEA), shared insights on the Coordinated Research Projects (CRPs): an IAEA tool to produce outputs by encouraging collaboration between various parties. He described the ongoing CRP on photonuclear data and photon strength functions, the TENDL $(\alpha, n)$ library improvements for safeguards, and IAEA's involvement in the Collaborative International Evaluated Library Organization (CIELO) on nuclear data evaluations for high-priority isotopes. Based on a 2016 planning meeting in Vienna, a new CRP for fission yields is expected to begin in 2019. 


\subsection{DISCUSSION PANEL}

Teresa Bailey of Lawrence Livermore National Laboratory discussed the process to measure, evaluate, and test nuclear data, along with quantification and propagation of uncertainties in nuclear models (Figure 2). She emphasized the need to be able to model systems from first principals where reality is not known.

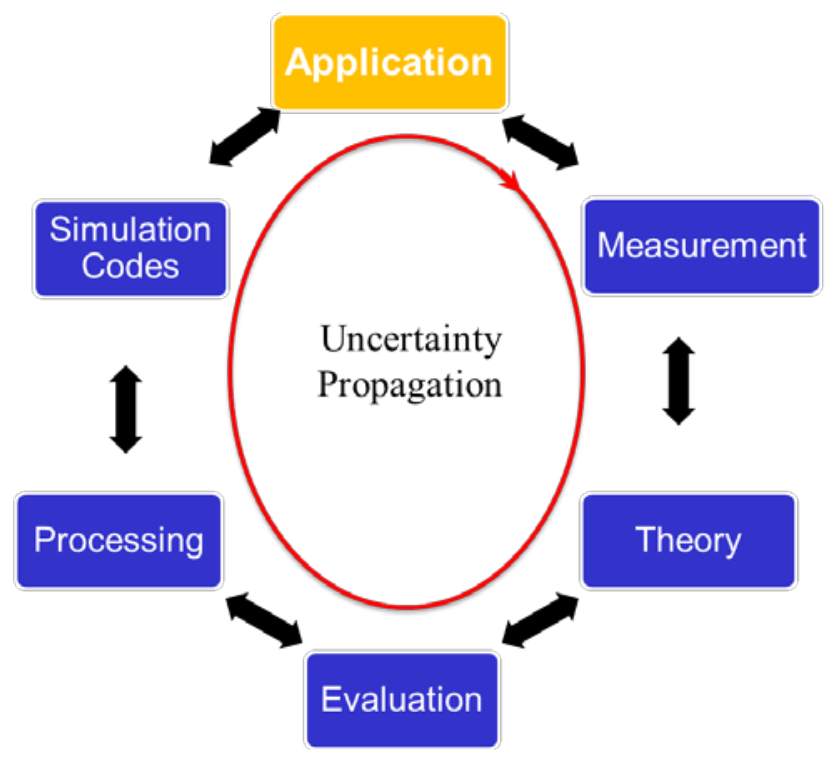

Figure 2. Illustration of the process to measure, evaluate and test nuclear data and the propagation of uncertainties.

Jerome Verbeke of Lawrence Livermore National Laboratory focused on the inability of simulation tools such as MCNP to adequately model the correlated neutron and gamma decay from neutron- and gammainduced fission and from inelastic scattering.

Lori Metz of Pacific Northwest National Laboratory described the impact of poor nuclear data on measurements for nuclear forensics, noting that production and decay data of Xe isotopes are of high importance, as are short-lived fission yields and specific activation products.

Brad Rearden of Oak Ridge National Laboratory discussed concepts related to uncertainty quantification and its complex impact on the utility of nuclear data. He pointed out that methods for quantifying and using uncertainties differ between applications, and the capability to propagate uncertainties within a simulation is important not only for determining nuclear data needs, but also for determining the total uncertainty in a predictive model.

David Brown of Brookhaven National Laboratory described the nuclear data pipeline to inform attendees of the steps needed to incorporate new measurements into the nuclear data libraries, including compilation, theory, evaluation, formatting, testing and validation. The nuclear data pipeline is illustrated in Figure 3. 


\section{The Nuclear Data Pipeline}

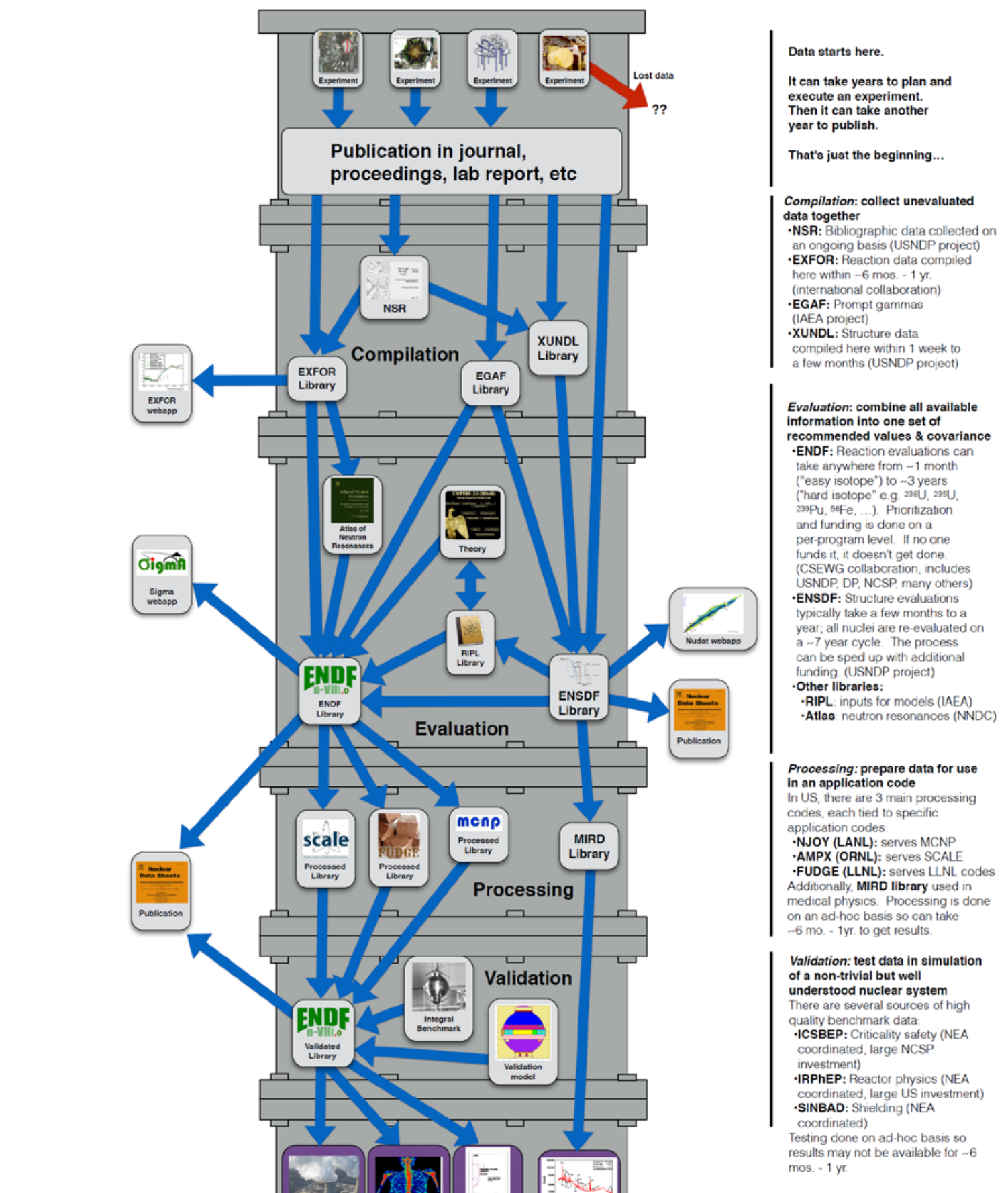

David Brown
24 Oct. 2017

BRDOMTRYEN
NATIONAL LABORATORY starts here.

can take years to plan an

Then an

year to publish.

That's just the beginning..

Compilation: collect unevaluated

ata together

GAF: Prompt gammas

(IAEA project)

a few months (USNDP project)

Evaluation: combine all available information into one set of

ENDF: Reaction evaluations can

take anywhere from $\sim 1$ month

"hard isotope" e g, 235U, $235 \mathrm{U}$,

${ }_{39 P u} 5 \% \mathrm{Fe}, \ldots$ ). Prionitization

(a) many others)

year; all nuclei are re-evaluated on

cyede. The process

Processing: prepare data for use

in an application code

ere are 3 main processing

pplication codes

NJOY (LANL): serves MCNP

-FUDGE (LLNL): serves LLNL codes

Additionally, MIRD library used in

$-6 \mathrm{mo}-1 \mathrm{yr}$. to get results.

there are several sources of high

Crick data

Cricality safety (NEA

mos. -1 yr

It can take years after an experiment is performed before the change appears in an application code

Figure 3. Illustration of the nuclear data pipeline. 


\section{DISCUSSION SESSION HIGHLIGHTS}

Four of the six half-day workshop increments were group discussion sessions. Each half-day increment consisted of three parallel discussion sessions for a total of 12 sessions over the course of the workshop. Each session was led by one or two session leads who are experts in the corresponding technical areas, and each session lead was supported by a note taker to capture comments of participants. Session leaders aimed to keep their discussions focused on nonproliferation and to develop specific needs and takeaways.

The summaries below discuss the goals and high-level contributions from each session. The detailed session summaries are provided in Appendix A and are primarily written by the session leads based on discussions and session notes. Participation in discussion was strong in all sessions, and the intent was to include all viewpoints in the summaries; therefore, recommended priorities and solutions may not be comprehensive and do not fully consider DNN R\&D mission priorities. Therefore, priorities in the DNN R\&D nuclear data strategic document will not necessarily be identical to the session notes.

\subsection{FISSION I: INDEPENDENT \& CUMULATIVE YIELDS}

\section{Session Leader: Patrick Talou, Los Alamos National Laboratory}

This session examined known deficiencies in fission yield data and discussed the required effort to reevaluate fission yields. For nonproliferation purposes, fission fragments represent the initial conditions that determine the emission of prompt and eventually $\beta$-delayed neutron and $\gamma$ emissions, which constitute signatures of specific nuclear materials. For nondestructive assay (NDA), delayed gamma-ray spectroscopy is often used to determine the presence of a given fission fragment species and infer the fissioning nucleus based on the intensity of specific gamma energies. For nuclear forensics, cumulative yields as a function of incident neutron energy are needed to identify the fuel and determine the neutron spectra that can be used to reconstruct and infer specific designs. For stockpile stewardship, cumulative yields are also needed to interpret historical data. For reactor antineutrino measurements, the fissioning nucleus and independent fission yields directly impact the antineutrino spectrum.

While the session covered experimental needs for fission yields, the thrust of the discussion was on the evaluation of existing data, and to a lesser extent, on theory and modeling needs. Because ENDF formats currently do not permit the storage of covariances for fission yield data, resolving this issue was deemed a high priority. The group acknowledged that some new experimental measurements for energy-dependent fission yield data may still be needed, although not much time was spent identifying specific isotopes and energy ranges in this regard. It may be beneficial to determine some of these needs as they arise during the evaluation process. The group identified its top priorities as the evaluations of fission yield data for

${ }^{235} \mathrm{U},{ }^{238} \mathrm{U}$, and ${ }^{239} \mathrm{Pu}$ over a range of energies. The need to address these data in the short term was driven in part by the opportunity afforded by the IAEA's upcoming CRP on fission yield evaluations.

\subsection{FISSION II: PROMPT NEUTRON AND GAMMA RAY EMISSIONS}

\section{Session Leader: Sara Pozzi, University of Michigan}

The goal of this session was to evaluate the state of nuclear data for prompt neutrons and gamma rays from fission and to prioritize needs for DNN R\&D. Prompt emissions from fission are pertinent to safeguards, emergency response, arms control, treaty verification, and other nonproliferation missions. Many safeguards and emergency response instruments use these signatures to detect and characterize fissile and fissionable materials, including neutron coincidence and multiplicity counters, as well as gamma spectroscopy systems. Tools developed more recently include neutron multiplicity counters which can measure prompt signatures such as neutron angular distributions and multiplicities at a short time 
scale (sub-ns). These instruments open the field to new applications and require knowledge of angular distributions, multiplicity-dependent energy distributions, and other higher order quantities. These data are currently deficient, even for important isotopes.

Participants identified two high-priority isotopes for spontaneous fission data, ${ }^{252} \mathrm{Cf}$ and ${ }^{240} \mathrm{Pu}$, and two for induced fission, ${ }^{235} \mathrm{U}$ and ${ }^{239} \mathrm{Pu}$. Other isotopes of interest included ${ }^{233} \mathrm{U},{ }^{238} \mathrm{U},{ }^{237} \mathrm{~Np},{ }^{238} \mathrm{Pu},{ }^{244} \mathrm{Cm}$, and ${ }^{250} \mathrm{Cf}$. Signatures of interest judged to be particularly important for these isotopes included energy spectra for prompt neutrons and gamma rays, number distributions (multiplicities), correlations between the number of neutrons and the energy distribution of the neutrons, angular correlations of the neutrons, and correlations between neutron and gamma ray emissions. The group also emphasized the development of relevant physics and transport codes such as CGMF, FREYA, MCNP6, MCNPX-PoliMi, and GEANT4. The codes need benchmark-quality data for code development and validation.

Overall, the group recommended conducting new experiments at existing facilities and using existing and new detection systems to capture the signatures of interest. Code development efforts should address improved models of fission emissions and validation using experimental data.

The session members emphasized the need to reexamine ${ }^{252} \mathrm{Cf}$ spontaneous fission measurement as a base standard before anything else.

\subsection{FISSION III: DECAY DATA}

\section{Session Leader: Elizabeth McCutchan, Brookhaven National Laboratory}

The goal of this session was to examine the status of decay data, particularly in the context of fission yields and beta-spectra data related to reactor antineutrino studies. Branching ratios dictate the relationship between cumulative and independent fission yields; however, cumulative fission yields are measured separately and maintained in an entirely separate database (ENDF) than branching ratios, which are in the Evaluated Nuclear Structure Data File (ENSDF) format. These data will support fission yield evaluations for DNN R\&D missions.

The group identified several specific isotopes as high priorities. Among the fission products, ${ }^{147} \mathrm{Nd}$ and branching ratios associated with Xe production were deemed especially important, with branching ratios for or associated with the production of ${ }^{133} \mathrm{Cs},{ }^{153} \mathrm{Eu},{ }^{141} \mathrm{Ce}$, and ${ }^{104} \mathrm{Ru}$ also being significant. The discussion emphasized branching ratios as the principal parameter of interest (or, in some cases, ratios of branching ratios). However, half-lives, gamma ray energies, decay heat energies, and the shape of the beta decay spectrum are also of interest. Uncertainty/sensitivity studies will be useful to determine the key pieces of nuclear data that impact the fission product of interest.

As an overall takeaway, it is important to communicate decay data priorities for evaluation to the NNDC. The group also emphasized value in developing a new application programming interface (API) for ENSDF to enable new parsing, search, and uncertainty capabilities.

\subsection{ACTINIDE CROSS SECTIONS}

\section{Session Leader: Susan Hogle, Oak Ridge National Laboratory}

The goal of this session was to address experimental measurement and theory for neutron absorption cross sections and decay of actinides. Several nonproliferation applications rely on the comprehensive understanding of the complex web of actinide isotopes interconnected by multiple neutron captures, $(n, 2 n)$ reactions, and subsequent decay. Relatively small uncertainties for individual cross sections can 
propagate into large uncertainties in overall accumulation and depletion rates, thereby restricting the utility of a particular method or measurement. A key challenge with this topic is the difficulty dealing with nuclides that may be short lived and/or difficult to isolate, thus presenting large gaps in many areas of the actinide web which may be difficult to fill through traditional measurement techniques.

Among the lighter actinides, the ${ }^{239} U(n, \gamma)$ and $(n, f)$ cross sections were perhaps the most emphasized among the group, although attendees also identified cross sections and decay data of the actinides ${ }^{235} \mathrm{U}$, ${ }^{237} \mathrm{U},{ }^{237} \mathrm{~Np},{ }^{238} \mathrm{~Np},{ }^{238} \mathrm{Pu},{ }^{236} \mathrm{Pu}$, and ${ }^{239} \mathrm{Pu}$. For applications requiring knowledge of heavier actinide accumulation rates, Cm was universally identified as the gateway for the production of heavier actinides, and ${ }^{243} \mathrm{Am}$, which leads to Cm production, was the most emphasized isotope, along with ${ }^{241} \mathrm{Am}$ to a lesser extent. Attendees also called for improved capabilities in structure-informed reaction modeling, particularly for $(n, \gamma)$ and $(n, 2 n)$ reactions, to better understand the properties of reactions in which target material would be very difficult to procure or measure.

Overall, the group advised developing a generalized data collection methodology, and as a demonstration case, applying it to a single isotope of high interest. Future proposals should emphasize evaluation and validation of new data rather than just experimentation.

\subsection{GAMMA-INDUCED REACTIONS}

\section{Session Leader: Brian Quiter, Lawrence Berkeley National Laboratory}

The goal of this session was to examine the nuclear data gaps in photonuclear reactions, such as photofission $(\gamma, f)$, photonuclear neutron release $(\gamma, n)$, and the observables from those reactions useful for nonproliferation applications. Areas of interest include active gamma-based interrogation techniques using bremsstrahlung or quasi-monoenergetic photon sources. Nuclear resonance fluorescence (NRF), may also be of use. Subcritical assembly experiments and forensics applications may also rely on highquality photonuclear data to understand their contribution amidst neutron-induced reactions. These parameters were deemed important not only by DNN R\&D, but also by DNDO for active interrogation of shipping containers.

This discussion mostly centered on needs related to ${ }^{235} \mathrm{U},{ }^{238} \mathrm{U}$, and ${ }^{239} \mathrm{Pu}$. For photofission, attendees desired improved data for incident neutron energies between 6 and $20 \mathrm{MeV}$, but especially between 6 and $9 \mathrm{MeV}$ for active interrogation. Neutron multiplicity was a high priority, including breakdown of prompt and delayed neutrons, with gamma multiplicity, angular distributions, fission product yields, and cross sections also being of interest. The discussion also addressed the need for better beam characterization and detector equipment.

\subsection{NEUTRON CAPTURE AND ASSOCIATED SPECTRA AND INELASTIC SCATTERING AND ASSOCIATED SPECTRA}

\section{Session Leader: Lee Bernstein, Lawrence Berkeley National Laboratory / University of California, Berkeley}

Sessions on capture gamma and associated spectra, as well as and inelastic scatter and associated spectra, were narrowly focused on addressing the observable $\gamma$-ray spectra and related nuclear data needs following $(n, \gamma)$ and (n,n' $\gamma$ ) to support active interrogation methods for nonproliferation missions. The need has come to the forefront only recently and was established based on input from NDA experts who described deficiencies when attempting to model and interpret gamma emission from neutron interactions. The goal of the session was to determine priority isotopes that require experiments, benchmarks, or evaluations and the process to provide discrete gamma energies and decay probabilities to 
the end user. The conclusions of previous discussions in the last two NDWG meetings (BNL/CSEWG $11 / 17$ and LANL 12/17) established the importance of (n,n' $\gamma$ ) cross sections on ${ }^{238} U,{ }^{235} U$ and ${ }^{239} \mathrm{Pu}$, and were described during the Nuclear Data Exchange Meeting on 4/14/16. Thus, the narrow focus for the $\operatorname{NDREW}(n, \gamma)$ session on the gamma emission spectrum was chosen in part to avoid overlap with other sessions which focused on the production/destruction aspects of neutron capture on actinides for forensics. Neutron capture cross sections on non-actinides has been an ongoing effort of the Nuclear Criticality Safety Program (NCSP), focusing primarily on structural materials and those used in nuclear materials processing. At higher energies, (n,n' $\gamma$ ) becomes increasingly important relative to (n $\gamma)$, and the quality and quantity of the data decrease significantly.

\section{7 (ALPHA,N) REACTIONS}

\section{Session Leader: Matt Devlin, Brookhaven National Laboratory}

The goal of this session was to examine $(\alpha, n)$ reactions and their neutron emissions which impact a number of nonproliferation applications. In some cases, $(\alpha, n)$ neutrons constitute the primary signal of interest, such as the measurement of outgoing neutrons, in order to estimate the quantity and enrichment of uranium hexafluoride. In other situations, $(\alpha, n)$ neutrons constitute a portion of the neutron background that needs to be subtracted from total measurements to determine some parameter of interest. Some of these parameters have large uncertainties which impact critical missions.

Most of the discussion emphasized reactions on ${ }^{19} \mathrm{~F},{ }^{17} \mathrm{O}$, and ${ }^{18} \mathrm{O}$ (with tangential mentions of Li, $\mathrm{C}$, and $\mathrm{Cl}$ ). Data are desired for the incident alpha energies ranging from slightly under $1 \mathrm{MeV}$ up to about $9 \mathrm{MeV}$. Reaction cross sections constituted the most thorough discussions, but outgoing neutron energy spectra were also considered a high priority. Moving forward, the group recommended designing benchmark studies and collecting thick-target data for the high-priority isotopes. In general, evaluations of recently obtained data were deemed to be a sensible near-term objective. Many applications use SOURCES4C [11] to calculate the passive neutron source term from $(\alpha, n)$ reactions. An update of this code is considered useful to support models of actinides in a fluoride or oxide matrix.

\subsection{DEVELOPMENT OF BENCHMARK EXERCISES}

\section{Session Leaders: Rian Bahran, Los Alamos National Laboratory, and Sean Stave, NNSA, Safeguards Technology}

The goal of this session was to start a conversation on benchmarks for nonproliferation missions that can be used to identify nuclear data deficiencies and validate existing and new differential data. The benchmarks must be specific to the application in many cases, but they can also be used to find deficiencies in the physics of the models. Uncertainty/sensitivity tools currently being developed for DNN R\&D or other offices should be used to analyze the benchmark data.

Session participants discussed lessons learned from the benchmark suites developed and used for the NCSP program in which nuclear data are validated with criticality experiments. Criticality benchmarks are not always appropriate for subcritical applications, and some examples were discussed.

Because benchmark experiments can be expensive, it was recommended that existing experiments be used that may be benchmark quality. Determining the appropriate procedure for selecting and analyzing these experiments will require further discussion. The consensus was that this is a useful path forward for DNN R\&D, but more work must be done to create a plan and methodology moving forward. 


\subsection{UNCERTAINTY, SENSITIVITY, \& COVARIANCE}

\section{Session Leader: Brad Rearden, Oak Ridge National Laboratory}

The session goal was to determine the current uncertainty, sensitivity, and covariance analysis capabilities required to (1) more readily determine nuclear data deficiencies and (2) understand the impact of nuclear data uncertainties on individual missions. Uncertainty quantification and sensitivity analyses are critical techniques for providing confidence margins on measurements and simulations for all nuclear applications, from radiation detection and shielding to nuclear weapons and reactors. Many tools are available or in development, but most require that new capabilities be developed to make them useful to the nuclear data community for DNN R\&D applications.

Primary needs include sensitivity and uncertainty quantification analysis tools for fixed source problems relevant to passive and active interrogation, correlated treatment of gammas and neutrons emitted from fission for uncertainty propagation in Monte Carlo simulations, reactor depletion calculations, actinide cross sections, and decay chain studies. Participants considered tools to support benchmark studies important. Broad scope, automated sensitivity and uncertainty quantification plug and play tools were desired.

\subsection{DATA PROCESSING \& TRANSPORT CODE NEEDS}

\section{Session Leaders: Brad Rearden, Oak Ridge National Laboratory and Teresa Bailey, Lawrence Livermore National Laboratory}

The session goal was to determine data processing and transport code needs for the proper use of existing and new types of nuclear data. Radiation transport is the fundamental particle physics framework at the core of all nuclear applications, from radiation detection and shielding to nuclear weapons and reactors. This framework supports a suite of simulation models and codes typically employed for proliferation and special nuclear material detection, tracking, and deterrence. In addition to neutron transport codes, codes are required to evaluate, process, and test nuclear data for use in transport models. All of these elements are required to provide the user with robust nuclear data.

Primary needs include treatment of particles emitted from fission including prompt neutron-neutron and neutron-gamma correlations, the timing of prompt emissions, and delayed emissions from decay of fission fragments. Temperature resolution for neutron thermal scattering law data, more complete photofission libraries and delayed emission treatments for active gamma interrogation, and capture gamma emission were all considered important. Furthermore, the user community expressed a desire for more validation, verification, and benchmarking of nuclear data across several application spaces.

The session members strongly emphasized the need for more complete ENDF libraries-perhaps employing theoretical models where needed-and improved linkage of ENDF to ENSDF.

\subsection{TARGETS, FACILITIES AND DETECTOR SYSTEM}

\section{Session Leaders: Jason Burke, Lawrence Livermore National Laboratory and Todd Bredeweg, Los Alamos National Laboratory}

Unlike other sessions, this session's goal was to address three largely distinct topics related to supporting capabilities for data improvements. Roughly equal time was allocated to each of the three topics. Targetry includes the production of necessary isotopes and the shaping of material to produce targets with geometries suitable for nuclear data experiments. Facilities include necessary capabilities such as neutron 
sources, reactors, critical assemblies, and others. Detector systems are used in conjunction with facilities to obtain the parameters of interest from a given experiment. These capabilities must be maintained to augment existing nuclear data with new measurements. Meeting the total requirements is more than a single organization can accomplish. Therefore, this session also explored the prospects of leveraging the resources of other organizations such as Office of Science facilities.

For targetry, attendees called attention to the cost of isotopes and the time required to produce targets. Thin target production capabilities for actinides is limited except for electrodeposition, which is not optimal for many experimental needs. There is also a shortage of enriched actinides to enable low uncertainty nuclear data experiments.

Top facility needs discussed included a setup specifically dedicated to neutron scattering measurements, along with work on the rabbit sample transfer system for the Device Assembly Facility (DAF) in Nevada. Detector needs included systems compatible with neutron scattering experiments, along with detection systems for gamma-induced fission measurements.

\section{DISCUSSION, CONCLUSIONS AND NEXT STEPS}

\subsection{CONSENSUS PRIORITIES}

The goal of each session was to not only identify and prioritize specific challenges for resolution, but also to discuss the effort required to get the data into the USNDP databases and available to the users. Participants were also asked to consider the past and current nuclear data projects within the United States and internationally and how to leverage and deconflict with these efforts. Many of these sessions were a first attempt at creating priorities and a recommended plan of work and will require additional discussion to determine how to best move forward. For example, the benchmarking session participants agreed that benchmarks are useful for determining nuclear data deficiencies and testing new data, but there was not a clear path forward at the end of the session.

There is consensus on the following cross-cutting priorities in nuclear data:

- Evaluate cumulative and independent fission yields for the "big three" isotopes: ${ }^{235} \mathrm{U},{ }^{238} \mathrm{U},{ }^{239} \mathrm{Pu}$, and some of the minor actinides.

- Conduct inelastic scattering on ${ }^{235} \mathrm{U},{ }^{238} \mathrm{U}$, and ${ }^{239} \mathrm{Pu}$ in the energy range of $1 \mathrm{keV}-3 \mathrm{MeV}$.

- Improve the knowledge of cross sections and decay properties of actinides within the network.

- Perform a SOURCES4C update to include measurements of the neutron emission energy spectrum from spontaneous fission and $(\alpha, n)$ reactions.

- Conduct application-specific benchmark experiments to test differential data, and apply uncertainty/sensitivity methods to determine nuclear data deficiencies

- Update the database infrastructure and modernize the methods used to produce evaluated data.

- Ensure that each new set of data includes covariance data and that the data can be processed for use in the codes.

\section{$5.2 \quad$ SURVEY INSIGHTS}

NDREW organizers developed a survey that was distributed shortly after the conclusion of the workshop. The survey collected information regarding attendees' overall opinions of the meeting, as well as their opinions on the venue, opportunities for collaboration, presentations, discussion sessions, and coordination/communication. The consensus was positive. In particular, attendees found it beneficial to start important discussions with many key participants and to develop collaborative opportunities. All 
respondents, including federal program managers, agreed that the workshop was useful and that they would attend a subsequent workshop.

The communication between various categories of participants is valuable. The workshop format enabled the following:

- Communication between the nuclear data community and program managers has shown great benefit in the past two years, providing valuable input to funding agencies on critical needs and best practices for nuclear data funding.

- Program managers were given the opportunity to share nuclear data priorities and ongoing projects.

- Universities were integrated into the discussions and gained a better understanding of how to prioritize their efforts.

- Discussions between users and producers allowed for a better understanding from both communities to guide work to fulfill mission needs.

- International collaborators were invited to participate and provide information on their priorities and ongoing projects.

It is important to continue these types of workshops to ensure that communication between communities continues. The reduction of stove-piping has led to useful collaborations, shared funding, deconflicting of planned funding, and an agreement on best practices so that all data can be used by all participants.

\subsection{NEXT STEPS}

Results from NDREW, combined with the outcomes from other meetings and follow-up discussions with data end users, will be used to develop a strategic document prioritizing nuclear data activities for DNN $\mathrm{R} \& \mathrm{D}$ over the next 5-10 years. It is anticipated that this document will be finalized in Fall 2018, and while there are no plans to make this document public, it will be used to inform decision-making for the office in subsequent years.

Many attendees expressed the desire to continue the discussions initiated at NDREW, possibly in the form of an annual workshop series. Specific implementation plans for this idea have not yet been finalized; however, a future workshop encompassing all applications will likely be spearheaded through DOE-SC-NP.

\section{ACKNOWLEDGMENTS}

Support for this workshop was provided by NNSA, DNN R\&D, under the auspices of the US Department of Energy at Oak Ridge National Laboratory under Contract DE-AC0500OR22725.

The work by staff from several laboratories was performed under the auspices of the US Department of Energy at Oak Ridge National Laboratory under Contract DE-AC0500OR22725, Los Alamos National Laboratory under Contract DE-AC52-06NA25396, Lawrence Livermore National Laboratory under Contract DE- AC52-07NA27344, Pacific Northwest National Laboratory under contract DE-AC0576RLO1830, Lawrence Berkeley National Laboratory under Contract DE-AC02-05CH11231, and Brookhaven National Laboratory under Contract DE-AC02-98CH10886. In addition, this work was funded in part by the Consortium for Verification Technology under Department of Energy National Nuclear Security Administration award number DE-NA0002534. 


\section{REFERENCES}

1. NNSA Nonproliferation Research and Development web page, https://nnsa.energy.gov/aboutus/ourprograms/nonproliferation/rd, Accessed 03/18.

2. D.A. Brown, M.B. Chadwick, R. Capote et al., "ENDF/B-VIII.0: The 8th Major Release of the Nuclear Reaction Data Library with CIELO-project Cross Sections, New Standards and Thermal Scattering Data,” Nucl. Data Sheets 148, 1-142 (2018).

3. Bernstein, L., et al. "Nuclear Data Needs and Capabilities for Applications," Proceedings of the Workshop on Nuclear Data Needs and Capabilities for Applications, Lawrence Berkeley National Laboratory, May 27-29, 2015. https://bang.berkeley.edu/events/ndnca/whitepaper.

4. Romano, C., “The Nuclear Data Working Group: Accomplishments and Future Plans,” Proceedings of the INMM 58th Annual Meeting, Indian Wells, CA, July 16-20, 2017.

5. Nuclear Data Interagency Working Group / Research Program, DOE National laboratory Announcement Number: LAB 17-1763, April 26, 2017. https://science.energy.gov/ /media/grants/pdf/lab-announcements/2017/LAB_17-1763

6. Talou, P., Kawano, T., Stetcu, I., Jaffke, P., and Rising, M.E., “CGMF: Event-by-Event Monte Carlo Simulations of Fission Fragment Decay,” to be submitted to Comp. Phys. Comm. (2018).

7. Verbeke, J. M., Randrup, J., and Vogt, R., "Fission Reaction Event Yield Algorithm FREYA 2.0.2,” Lawrence Livermore National Laboratory, LLNL-JRNL-728890 (2017).

8. Goorley,T., et al. “Initial MCNP6 Release Overview - MCNP6 version 1.0,” Los Alamos National Laboratory, Los Alamos, NM, LA-UR-13-22934 (2013).

9. Nuclear Data Interagency Working Group / Research Program, DOE National Laboratory Announcement Number: LAB 18-1903, March 26, 2018. https://science.energy.gov/ /media/grants/pdf/lab-announcements/2018/LAB 18-1903.pdf

10. Arjan Koning, Stephane Hilaire, and Stephane Goriely, TALYS-1.6, A Nuclear Reaction Program, Nuclear Research and Consultancy Group, Westerduinweg 3, Petten, the Netherlands (2013).

11. Wilson, William B., Perry, Robert T., Shores, Erik F., Charlton, William S., Parish, Theodore A., Estes, Guy P., Brown, Thomas H., Arthur, Edward Dana, Bozoian, Michael, England, T.R., Madland, D.G., \& Stewart, James E. (Jan 2002). SOURCES 4C: a code for calculating $(\alpha, n)$, spontaneous fission, and delayed neutron sources and spectra (LA-UR--02-1839). 
APPENDIX A. DETAILED SESSION SUMMARIES AND NOTES 



\section{A.1 Fission I: Independent and Cumulative Fission Yields}

\section{Session Leader: Patrick Talou, LANL}

This session focused on evaluation of the independent and cumulative fission yields to support multiple nonproliferation missions, including post-detonation forensics, NDA measurements, and reactor fission product content. This topic is important due to the known fission yield uncertainties, as well as inconsistencies between the decay data and the cumulative fission yields. The goal of the session was to determine a path forward to a new evaluation including required experiments, evaluation tool development, and workforce needs.

\section{A.1.1 Background}

Scientific interest is focused on the part of the fission process that occurs past the scission point, or the point of separation between the two fragments. The newly formed fission fragments emit prompt neutrons and gamma rays within $\sim 10^{-14} \mathrm{~s}$, with some prompt gammas coming later, within $\sim 1 \mathrm{~ms}$, due to the presence of isomers. The yields of the fission fragments in mass and charge, $\mathrm{Y}(\mathrm{A}, \mathrm{Z})$, after prompt neutron emissions, are called independent fission yields (IFYs). Eventually, the neutron-rich fragments undergo gamma-decay when a neutron transforms into a proton, an electron, and an antineutrino. Following gamma-decay, more neutrons and gamma rays can be emitted. Those are called gamma-delayed emissions. The final fission product yields are called cumulative fission yields (CFYs). This is illustrated in Figure A-1 below:

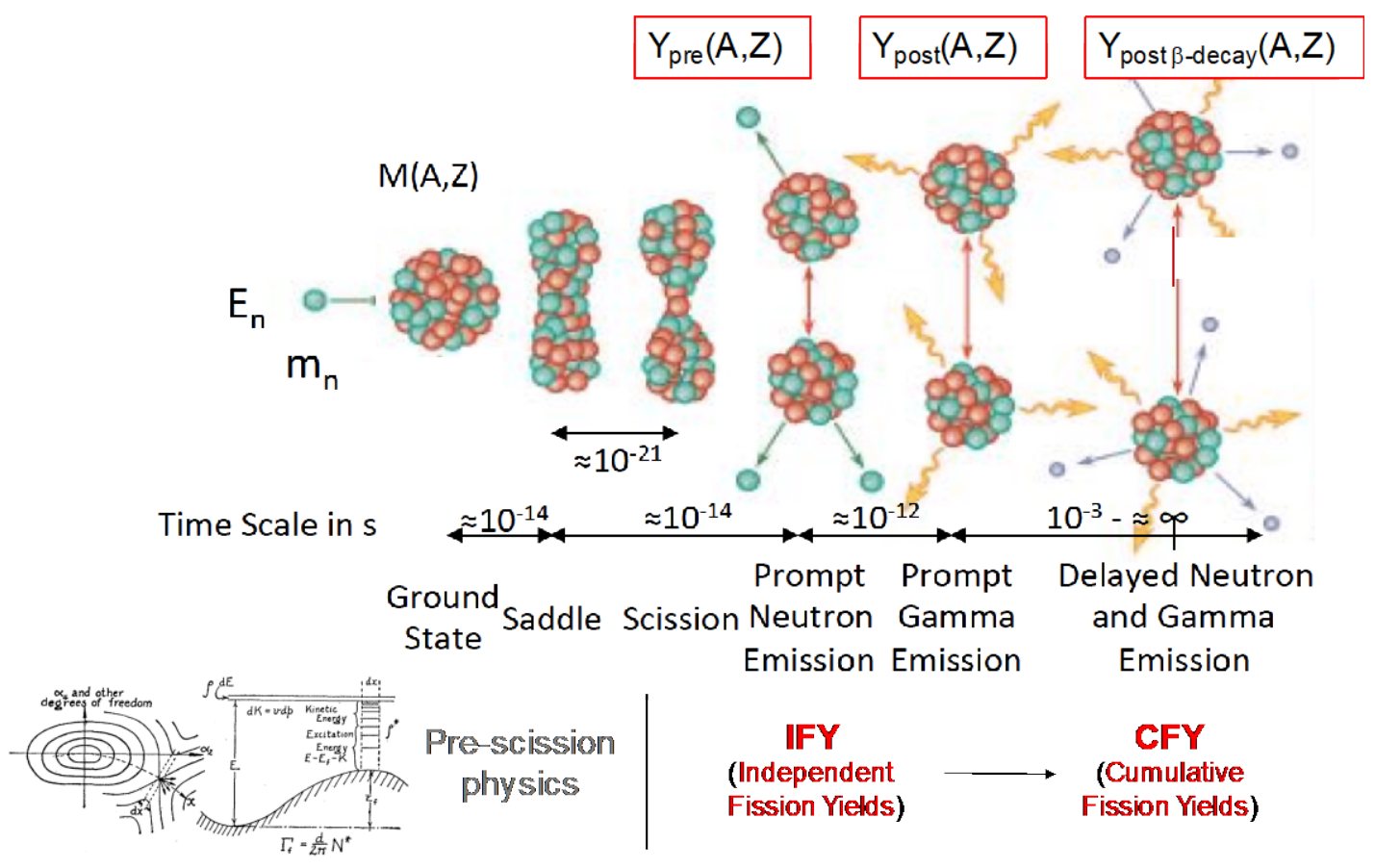

Figure A-1. Schematic of the fission process.

A consistent, coherent description of this decay chain has yet to be developed for nuclear data evaluation purposes, although all the necessary pieces of physics theories, experimental data, and model codes are 
available to develop the theory. However, many important, unanswered questions remain regarding the details of this decay chain. Also, the correlations expected in a coherent description of the complete process present significant challenges and opportunities.

\section{A.1.2 Significance}

Fission yields are important for a variety of applications and for basic scientific understanding of the fission process and many-body nuclear physics.

For nonproliferation purposes, fission fragments represent the initial conditions that determine the emission of prompt and eventually $\beta$-delayed neutron and $\gamma$ emissions. These emissions constitute signatures of specific nuclear materials. A consistent representation of IFY and CFY puts indirect but stringent constraints on the characteristics (multiplicity, energy, angle) of those light particle emissions. Gamma-ray spectroscopy is often used to determine the presence of a given fission fragment species and to infer its yield based on the intensity of specific $\gamma$ lines or double- and triple-coincidence $\gamma$ gates.

For nuclear forensics, CFYs are needed to identify the fuel and determine the neutron spectra that can be used to reconstruct and infer specific designs. For stockpile stewardship, CFYs are also needed to interpret historical data. For both applications, CFYs are needed as a function of incident neutron energy and isotope. IFYs and CFYs represent snapshots of the fission process at different times in the decay chain, and their correct description relies on a good understanding of the neutron and $\gamma$ emission probabilities and energy spectra, as well as reliable information on the structure of neutron-rich nuclei. In astrophysics, fission recycling has been shown to have a significant impact on predicted solar abundances, depending in large part on the ratio of symmetric vs asymmetric fission yields produced in a particular neutron environment. CFYs from reactor fuel and plutonium isotopes are also needed to accurately model the antineutrino spectrum, which in turn is important to interpret the antineutrino anomaly. Isomeric ratios and $\beta$ spectrum shapes of a few important contributors also need to be known accurately.

For nuclear energy and nuclear waste management purposes, fission yields are needed for decay heat, shielding, dosimetry, fuel handling and safe waste disposal. They are also critical to properly perform a fission product inventory at each stage of the nuclear fuel cycle. The development of advanced reactor and waste transmutation concepts requires accurate fission yield data. This is also true for existing reactors that must follow increasingly stringent safety standards.

Other applications include safeguards for nuclear reactor monitoring, medical applications for radioisotope production, etc.

\section{A.1.3 Existing and Recent Projects}

Many efforts related to fission yield are already underway, funded through different sponsors, as summarized here.

\section{A.1.3.1 Experiments}

- Since 1994, new 2E experiments in which the kinetic energies of the two complementary fragments are measured have been performed in the US (RPI, LLNL), Europe, and Japan. With the help of IAEA consultants, the NNDC, is working on providing a complete, clean compilation of the available experimental data. $2 \mathrm{E}$ experiments are limited by mass resolution when compared to the $2 \mathrm{E}-2 \mathrm{~V}$ experiments. The measured values are of the post-neutron emission masses and require the knowledge of the average neutron multiplicity as a function of mass, $v_{\text {avg }}(\mathrm{A})$, to correct the observed masses for neutron emission to obtain pre-neutron emission masses. Such 
neutron data are rarely available beyond ${ }^{252} \mathrm{Cf}(\mathrm{sf})$ and thermal neutron-induced fission reactions on major actinides, increasing uncertainties at higher incident energies. The benefit of these experiments is their higher efficiency, allowing for thin targets which improve resolution, and the ability to measure small quantities of the target actinide.

- The SPIDER experimental setup at the Los Alamos Neutron Science Center (LANSCE) is dedicated to the measurement of fission fragments in a $2 \mathrm{E}-2 \mathrm{~V}$ setup in which the two fragment kinetic energies and velocities are measured simultaneously. Preliminary studies on ${ }^{252} \mathrm{Cf}(\mathrm{sf})$ have shown that 1 mass unit resolution is achievable. Similar setups-COSI FAN TUTTE at ILL, France and VERDI at JRC-Geel, Belgium - have shown similar results. Extending such measurements to neutron-induced fission reactions in the fast energy range is certainly the greatest challenge to overcome. To achieve this, a significant upgrade of the instrument, MEGASPIDER, is being proposed.

- The SOFIA Coulomb excitation (Coulex) fission experiments at GSI have provided a vast amount of fission yield data, with impressive accuracy in mass and charge (dA and dZ resolutions better than one unit). However, these are obtained over a rather broad spectrum of excitation energies whose average is about $11 \mathrm{MeV}$. The data are not directly usable for an evaluation of the fission yields, but they can be used to validate and constrain them. Some preliminary results have been published already, but many more are being analyzed.

- Recent measurements of the average total kinetic energy (TKE) of the fission fragments have been performed at the LANSCE WNR facility for energy from a few hundred keV to $200 \mathrm{MeV}$. The average TKE is not flat but is mostly decreasing with excitation energy. These new data for ${ }^{239} \mathrm{Pu},{ }^{235} \mathrm{U}$ and ${ }^{238} \mathrm{U}$ have been used for a new evaluation in the new ENDF/B-VIII library. TKE is not directly present in fission yield evaluations but is an important and very sensitive input to codes that compute neutron and gamma emissions from the fission fragments. The observed increase of TKE at low incident energy (below $1 \mathrm{MeV}$ ) must be confirmed and is the current subject of theoretical studies.

- The surrogate reaction technique is used by LLNL at Texas A\&M to measure the ${ }^{239} \mathrm{Pu}$ and ${ }^{241} \mathrm{Pu}$ prompt fission neutron multiplicity (average and distribution) as a function of equivalent incident neutron energy from $100 \mathrm{keV}$ to $20 \mathrm{MeV}$. while those data are not direct measurements of fission yields and the uncertainties are not well determined, they do impact the theoretical and modeling tools that have been developed to correlate those quantities.

- As part of the Nuclear Forensics program, LANL and PNNL have been performing R-value measurements of fission products produced on various critical assemblies like Flattop at the National Criticality Experiments Research Center (NCERC) at the Nevada National Security Site. $\mathrm{R}$-values are simply ratio of fission yields that eliminate some of the difficulties in measuring absolute values. Activation measurements of various irradiated samples have been performed, and a new measurement campaign using new fission chambers will continue in the next few years.

- In the last few years, LLNL and LANL activation measurements at Triangle Universities Nuclear Laboratory (TUNL) have provided invaluable data on the incident energy dependence of CFYs that support re-evaluation of ${ }^{239} \mathrm{Pu}$ fission yields by Kawano and Chadwick at $2 \mathrm{MeV}$. The more complicated behavior between 0.5 and $15 \mathrm{MeV}$ represents a challenge for theoretical calculations. A complementary measurement is planned with thermal neutrons at MIT. A new experimental effort at TUNL will also test the Bohr hypothesis of independence between entrance and outgoing channels in a compound reaction by comparing fission yields in the ${ }^{239} \mathrm{Pu}(\mathrm{n}, \mathrm{f})$ and ${ }^{240} \mathrm{Pu}(\mathrm{gamma}, \mathrm{f})$ reactions that lead to the same fissioning system, at least in mass and charge. The photo-fission experiment will use the HIGS facility at TUNL.

- Two new projects started under Office of Science funding at the CARIBU facility. The first one uses the CARIBU facility and associated detector setups to measure fission product properties, isomeric yield ratios, gamma-ray decay branching ratios, and gamma-delayed neutron emission 
properties. The second aims at improving antineutrino spectrum simulations by measuring gamma-decay data using Gammasphere at CARIBU.

\section{A.1.3.2 Theory}

Theoretical studies of fission fragment yields have seen major breakthroughs in the last decade or so. Below is a summary of major ongoing efforts:

- Fission fragment charge and mass distributions have now been performed using Monte Carlo random walks using semi-classical macro-micro calculations of the potential energy surfaces (PESs) that describe the shapes of the heavy nucleus on its way to fission. The underlying phenomenological model of the quantum-deformed liquid drop has been used very successfully over the years to compute nuclear masses, fission barrier heights, and $\gamma$-decay strength functions. The recent extension to compute fission fragment distributions has been able to reproduce major trends observed in the evolution of the symmetric and asymmetric modes of fission across the nuclear chart. Recent theoretical studies have shown that such a model can be used to predict important prompt neutron and gamma observables relatively well, although some specific prompt neutron quantities will require optimized and tuned versions of those codes to reach a higher accuracy as required by the applications. Because such computations are relatively fast, this model is ideal for parameter optimization purposes.

- More fundamental calculations of the fission process based on constrained and unconstrained microscopic theories using nucleon-nucleon forces have also expanded rapidly in the last decade thanks to exploding computer capabilities. Time-dependent constrained Hartree-Fock (TDHF) calculations coupled with Langevin simulations have been used to infer fission yields produced in the spontaneous fission of ${ }^{240} \mathrm{Pu}$.

- Finally, the most unconstrained approach to this many-body quantum mechanical problem (TD-SLDA) starts to provide important answers (and to present more questions) to guide the more phenomenological models. Important questions concern the sharing of the total excitation energy between the two complementary fragments near scission and the production of angular momentum in both fragments. Those two components represent the most important initial conditions that in large part determine the details of the following decay sequence of prompt neutron and gamma emissions.

- One of the goals of the FIRE project is to guide fast macro-micro calculations of fission yields with more fundamental, but also more computationally expensive, microscopic calculations, providing efficient, scientifically sound predictions of fission yields for the actinide region and for astrophysics input (fission recycling).

\section{A.1.3.3 Models \& Evaluations}

- The latest fission yield evaluations in the US ENDF/B-VIII library correspond to those performed by England and Rider in Los Alamos in 1994. The library contains a total of 40 fissioning systems - 9 for spontaneous fission and 31 for neutron-induced fission reactions. For the Big Three- ${ }^{235} \mathrm{U},{ }^{238} \mathrm{U}$ and ${ }^{239} \mathrm{Pu}$ - three evaluations are given at three energies: thermal, fast (fission spectrum energies), and high (14 MeV). More recently, Kawano and Chadwick added a fourth incident energy point at $2.0 \mathrm{MeV}$ to the ${ }^{239} \mathrm{Pu}$ fission yield evaluation. In Europe, Robert Mills (UK) has been revisiting fission yields for the European JEFF library.

- At LANL, in collaboration with Japanese colleagues at Tokyo Institute of Technology, a new model has been developed to compute the decay of fission fragment yields by prompt neutron and gamma-ray emissions, followed by gamma-decay and gamma-delayed emissions. A preliminary study of low-energy fission of $\mathrm{n}+{ }^{235} \mathrm{U}$ has been reported in a publication submitted to the Journal of Nuclear Science and Technology (JNST). 
- In Europe, the GEF code, developed by Schmidt and Jurado, has gained popularity for performing similar calculations, from fission fragments to IFYs to CFYs. It is now being used as part of the JEFF evaluation efforts.

\section{A.1.4 Current Needs}

\section{A.1.4.1 Experimental Needs}

- Accurate, energy-dependent fission fragment yields and CFYs are needed for important actinides. The work with MEGA-SPIDER, at TUNL, and at NCERC should be continued to provide important validation and calibration points to any evaluation effort.

- Efforts of fission neutron and gamma-ray spectrum measurements that link the fission fragments and products should be continued, as illustrated in the figure above.

- Fission yield measurements at HIGS should also be continued.

- The sources of experimental uncertainties and correlations should be reported in detail—not as a single covariance matrix, but as a report on distinct sources of errors, calibrations, and statistical vs. systematics components. The incorporation of a USNDP representative in the nuclear data experiments will ensure the appropriate rigor when determining reported uncertainties.

\section{A.1.4.2 Theory Needs}

While it is not expected that fission theories will be able to have a direct impact on applications in the near future, their indirect influence can be very important. Of particular interest is the evolution of the initial fission fragment distributions in mass, charge, kinetic energy, excitation energy, and angular momentum as a function of incident neutron energy, as they strongly influence the following neutron and gamma emissions. Another quantity of interest is how many neutrons are possibly emitted before scission occurs, the so-called pre-scission and scission neutrons, and their energy spectrum and angular distribution. Support for more fundamental models of fission is important to guide the scientifically sound development of simplified phenomenological models that can be deployed for larger scale calculations directly usable for evaluation purposes.

In this regard, increased support for microscopic (LLNL, UW, MSU) and macro-micro (LANL) calculations is needed. Continuing support for codes that can compute the prompt and $\gamma$-delayed neutron and gamma emissions is important to link fission fragments to IFYs to CFYs and to provide a consistent picture of the post-scission process that will lead to realistic uncertainties and correlations.

\section{A.1.4.3 Modeling / Computation Needs}

There is a clear need for the development of a modeling and evaluation code that can compute IFYs, CFYs and prompt and beta-delayed neutron and gamma emissions consistently. The evaluated decay data should also remain consistent with any new evaluation of the fission yields. There should be consideration of how these data remain linked so that any evaluation of decay data is linked to the evaluated fission yield data.

Uncertainty quantification (UQ) tools should be developed as part of this physics modeling effort so that fission yield covariance matrices are evaluated concurrently to the evaluated files. Such UQ tools should consider experimental and theoretical knowledge of the yields and follow the evaluation procedure as closely as possible. Sensitivity calculations should be performed with new modeling tools (i.e., ORSEN developed at ORNL) to assess the most valuable nuclear data required to reduce uncertainties in certain cumulative yields. Phenomenological but well-justified physics models should be further developed, in 
coordination with experimental and theoretical developments, to create a state-of-the-art evaluated library of fission yields.

\section{A.1.4.4 Evaluation and Data Format Needs}

The evaluated files present in ENDF/B-VIII contain some uncertainties but no covariances, as the current ENDF format does not allow for such information to be compiled. This is a clear data format need that should be addressed by the CSEWG Format Committee acting on proposed extensions of the ENDF and GNDS formats.

The evaluated IFYs and CFYs are not consistent with the current nuclear structure data. They will remain mostly inconsistent as long as the release cycles of the two sublibraries remain on two separate evaluation tracks. It is an important aspect of any new evaluation effort that should be addressed early on, and any new evaluation should address this question of consistency.

More generally, it is important to realize that although IFYs, CFYs, prompt and $\gamma$-delayed neutrons and $\gamma$ rays and decay data are physically correlated, the data included in the evaluated libraries are not. This is because they are all evaluated independently using models that are developed for only one specific aspect of the fission process. Recently, new physics models have been developed to consistently describe the post-scission phases of the fission process. There are now physics models that can calculate the fission fragments created right after the scission point, compute their decay by emission of prompt neutrons and $\gamma$ rays, calculate the probability for their further $\gamma$ decay, followed again by a sequence of $\gamma$-delayed neutron and $\gamma$-ray emissions.

\section{A.1.4.5 Workforce/Infrastructure Needs}

Some of the knowledge on fission yields in the United States has disappeared with the last evaluators- $\mathrm{T}$. R. England, B. F. Rider, and W. B. Wilson-who focused on this important topic. Given the critical application needs for new fission yield evaluations, any effort to rebuild this workforce and drastically improve the modeling capabilities in the United States is a high priority. This should be developed as a multi-lab capability incorporating qualified students.

\section{A.1.5 High-Level Goals}

The high-level goals and priorities that emerged during the discussion session are summarized here. The exact order of priorities depends on the specific application in mind; however, the highest priorities identified during the workshop appear in bold.

- Evaluations

o New evaluations with energy dependence from thermal up to $20 \mathrm{MeV}$

o Priority isotopes:

- $\quad(n, f):{ }^{233,235,238} \mathrm{U},{ }^{239} \mathrm{Pu},{ }^{241} \mathrm{Pu},{ }^{237} \mathrm{~Np}$

- Spontaneous fission: ${ }^{252} \mathrm{Cf},{ }^{240} \mathrm{Pu},{ }^{238} \mathrm{U}$

- $\quad$ Photofission in the energy range of 6-9 MeV (covered under another session)

o Specific isotopic yields and isomeric ratios (strongly depends on the application)

o Need of new ENDF/GNDS format for fission yield covariances

o A comprehensive, consistent, optimized, well-documented evaluation tool developed and shared by the community; correlations and uncertainties built-in directly in the evaluation process

- Experiments: 
o Compilation, clean up, and organization of fission yield experimental data (NNDC already started a project with IAEA consultants)

o Measurements of prompt and beta-delayed fission neutrons and gamma rays (addressed in a separate session)

o Decay data of fission fragments (addressed in a separate session)

o Fission product projects at CARIBU

o R-value measurements

0 Activation measurements

o Nu-bar and $\mathrm{P}(\mathrm{nu})$ measurements on surrogate reactions

$0 \quad$ Photofission and (n,f) experiments

o Leverage SOFIA@GSI work in fission “Coulex” in inverse kinematics

- Theory

o Computation of initial conditions of fission fragments in excitation energy and spin as a function of incident neutron energy

o Emission of pre-scission neutrons, their multiplicity, and spectrum

0 Understanding of energy dependence of fission yields in (A,TKE)

o FIRE (currently funded) work at the intersection of phenomenological and more fundamental theories of fission yields

o Calculation of $\mathrm{P}(\mathrm{n})$ values

- Validation, Benchmarking and Applications

o Flattop and Godiva experiments

o Radchem analysis on dissolved targets and beta counting

o Feedback from the FIRE work in astrophysical r-process

o Irradiation work for short-lived yields

o Experimental and simulation studies of complex "blobs" of Pu and other objects

\section{A.2 Fission II: Prompt Gammas and Neutrons}

\section{Session Leader: Sara Pozzi, University of Michigan}

This session was motivated by the need to understand and model the correlated neutron and gamma emissions from fission including the energy spectra and angle of emission to support NDA techniques. Recent work has incorporated FREYA and CGMF into MCNP to support models of fission gammas and neutrons, but there are still improvements required in the data. The goal of the "Fission II: Prompt Gammas and Neutrons” session was to evaluate the state of the nuclear data for prompt neutrons and gamma rays from fission and prioritize needs based on current DNN R\&D priorities. Prompt emissions from fission have application in nuclear safeguards, nonproliferation, emergency response, arms control, and forensics.

\section{A.2.1 Background}

Prompt emissions from fission are essential in the detection and characterization of special nuclear materials (uranium enriched in ${ }^{235} \mathrm{U}$ and ${ }^{239} \mathrm{Pu}$ ). There are many safeguards and emergency response instruments that make use of these signatures. These include neutron coincidence and multiplicity counters, gamma spectroscopy systems, and activation analysis. While neutron multiplicity detectors have been in use for several decades now, there are a number of newly developed systems that make use of new detection materials. These include neutron multiplicity counters at LLNL and UM that can measure prompt signatures such as neutron angular distributions and multiplicities at a short time scale (sub-ns). These instruments open the field to new applications, and they require knowledge of angular distributions, multiplicity-dependent energy distributions, and other higher-order quantities. These data are currently 
missing in our databases, even for important isotopes. Thus, the members of the session identified several needs in nuclear data that should be addressed in future efforts.

\section{A.2.2 Needs}

Nuclear data needs include studying the prompt emissions from spontaneous fission and induced fission (both neutron- and photon-induced).

A. High priority isotopes

i. Spontaneous fission:
1. ${ }^{252} \mathrm{Cf}$

2. ${ }^{240} \mathrm{Pu}$

ii. Induced fission (neutron-induced and photofission):

1. ${ }^{235} \mathrm{U}$

2. ${ }^{239} \mathrm{Pu}$

iii. Other isotopes of interest include

1. ${ }^{233} \mathrm{U}$

a. n-induced fission and spontaneous fission parameters

2. ${ }^{238} \mathrm{U}$

a. n-induced fission, photofission, and spontaneous fission parameters

3. ${ }^{237} \mathrm{~Np}$

a. n-induced fission and spontaneous fission parameters

4. ${ }^{238} \mathrm{Pu}$

a. Spontaneous fission

5. ${ }^{244} \mathrm{Cm}$

a. Spontaneous fission

b. Important for safeguards / spent fuel

6. ${ }^{250} \mathrm{Cf}$

a. Spontaneous fission

b. Older ${ }^{252} \mathrm{Cf}$ sources are commonly "contaminated" with ${ }^{250} \mathrm{Cf0}$

B. Signatures of interest (prompt) from these isotopes include:

i. Energy spectra for prompt neutrons and gamma rays

ii. Number distributions (multiplicities)

1. Point source vs. distributed source

2. Moments

iii. Correlations between the number of neutrons and the energy distribution of the neutrons

iv. Angular correlations of the neutrons

v. Correlations between neutron and gamma ray emissions

C. Other signatures

i. Gamma ray multiplicity spectrum, particularly for photofission

ii. Time of emission

iii. Timescale of prompt (before beta decay) gammas (more important)

iv. Timescale of delayed neutrons (less important)

v. Incident neutron energy effect on the multiplicity distribution (high importance for ${ }^{235} \mathrm{U}$ and ${ }^{239} \mathrm{Pu}$ )

D. Additional nuances

i. Useful combinations/correlations of the signatures

ii. Gamma-ray spectrum vs gamma-ray multiplicity

iii. General lack of photofission data below $10 \mathrm{MeV}$ 


\section{A.2.3 Physics and Transport Codes}

Several physics and transport codes are used in this mission space. These include CGMF, FREYA, MCNP6, MCNPX-PoliMi, and GEANT4. The codes require benchmark-quality data for code development and validation. Validated codes with full descriptions of the prompt emissions from fission are valuable for applications because they can be used to predict the response of new and existing detection systems. Code development is needed in the areas of correlations between prompt neutrons and gamma rays, energy and angular correlations of prompt neutrons, and time emission distribution of prompt gamma rays. Photofission is still quite lacking, with errors of $40 \%$ or more. Any improvements in this area would be beneficial.

\section{A.2.4 Recommended actions}

Covering the data needs for the prompt emissions from fission include conducting new experiments at existing facilities and using both existing and new detection systems to capture the signatures of interest. Nuclear data needs are listed previously. Benchmark experiments will be useful where results can be shared with the community for inclusion in ENDF evaluations. In code development, efforts should address improved models of fission emissions and validation using experimental data. Validation of FREYA and CGMF as incorporated in MCNP using the previously mentioned benchmark experiments should be a priority. Because of its correlated data capabilities and fast processing, FREYA has been used in many applications with relatively good results and should be supported further. Facilities available to support experiments are listed below:

\section{A.2.4.1 Facilities in the United States}

1. LANL/LANSCE (Chi-Nu, DANCE, NEUANCE)

a. NEUANCE can get close to source of $1 \mathrm{MeV}$ (around which issues with scattering occur)

b. Lujan starts to run out of neutrons above $\sim 0.5 \mathrm{MeV}$

2. RPI

3. University of Michigan (UM): $9 \mathrm{MeV}$ electron LINAC that UM plans to use for some photofission studies

4. Idaho State

a. Can handle photofission (detection system has been dismantled)

5. Texas A\&M

a. 3.7-ton liquid scintillator to measure neutron emission

b. Could include a target in the facility for spontaneous fission, but it is typically used for induced fission

c. $80-90 \%$ efficiency for single-neutron

d. Scintillator will require replacement after $\sim 10$ years

e. Does NOT collect neutron energy / spectrum info

6. VANDLE array at ORNL, plastic scintillator

a. Used mostly for delayed neutrons but can also be used for prompt neutrons

7. Facilities at or near TUNL

a. Van De Graaf generator which enables tuning of the neutron beam. For deuterium or tritium, can go up to $1 \mathrm{MeV}$. Resolution is at $2 \%$

b. Free electron laser (2 MeV Compton at Duke) 


\section{A.2.4.2 International Facilities}

1. French ionization chamber (CEA)

a. Fission chamber (can accommodate larger samples than other methods)

\section{A.3 Fission III: Decay Data}

\section{Session Lead: Elizabeth McCutchan, National Nuclear Data Center, Brookhaven National Laboratory}

This NDREW session was originally designed to address decay data needs relevant to fission yield measurements and evaluations, so it is included as one of three sessions relating specifically to fission yields. However, the significance of decay data to other aspects of the DNN mission was quickly recognized, so the scope of the session was expanded to include the topics of reactor antineutrino spectra, as well as forensics and safeguards. The session frequently took detours to discuss user requests for better tools to interact with the decay data available in the current databases.

The only comprehensive source of decay data in the world is the ENSDF maintained at the National Nuclear Data Center at Brookhaven National Laboratory. ENSDF is updated monthly, allowing for results to be quickly disseminated to the user community. With every release of the ENDF library, a decay data sublibrary is included and updated, where the ENSDF data are converted into the ENDF format, and additional information like x-rays and Auger electrons are incorporated.

Three efforts were presented here which have or will soon greatly enhance the quality of decay data in ENSDF. At ORNL, the MTAS total absorption spectrometer has measured the beta-feedings for a total of 77 high priority nuclides, which are important for decay heat determinations and calculation of reactor antineutrino spectra. Some results have already been published, and the remainder of the measured nuclei are currently under analysis. Two new proposals have been funded through a recent Nuclear Data Interagency FOA. One proposed effort is to perform a combination of total absorption and discrete gamma ray spectroscopy on approximately 30 nuclei relevant to the calculation of antineutrino spectra from a nuclear reactor. The other will address several topics, including measurements of fission product isomer-to-g.s. ratios, beta-delayed gamma-ray branching ratios, beta-delayed neutron emission probabilities, and the beta spectra of fission fragments. Both proposals will use beams from the ${ }^{252} \mathrm{Cf}$ CARIBU source at Argonne National Laboratory.

To undertake a new fission yield evaluation, accurate knowledge of the fission fragments' basic decay properties is required. A new fission yield evaluation will need to convert measured independent yields to cumulative fission yields. To do this, the delayed neutron branching ratios of all the fission fragments are required, as well as the spin and excitation energy of longer-lived isomeric levels. There is currently an IAEA CRP on beta-delayed neutron properties of fission fragments; the final report is due in late 2018. This document carefully compiles and evaluates all beta-delayed neutron emission probabilities and provides guidance on where data are discrepant or lacking. Thus, this document will be an excellent resource for planning new experimental campaigns to address the gaps in data needed to take us from independent to cumulative yields. Future fission yield measurements are also underway or planned. These measurements irradiate samples with neutrons and then deduce the fission yields from the gamma-ray emissions of the fragments. This technique is only successful if the data on the delayed gamma-ray branching ratios are accurate. However, the decay data in the databases are either quite outdated, particularly for the shorter-lived fragments, or they suffer from the Pandemonium effect. Thus, new fission yield measurements will require a careful evaluation of the decay data involved, and new measurements of branching ratios may also be required. 
For forensics and safeguards, gamma-ray branching ratios are the essential need for decay data. Xe isotopes and ${ }^{133} \mathrm{Cs}$ were mentioned by several participants for forensics. The quality of the data in ENSDF should be assessed; subsequent measurements may be needed, as these isotopes are routinely used. High quality data on ${ }^{147} \mathrm{Nd}$ and ${ }^{153} \mathrm{Sm}$ are required for safeguards. ${ }^{104} \mathrm{Ru}, \mathrm{Rh},{ }^{141} \mathrm{Ce}$, were also mentioned. For actinides, a new proposal funded by the interagency ND FOA will be studying Np isotopes, which will also require decay data. The Np decay data were also highlighted as a need in the Berkeley ND Workshop, with highest priority given to ${ }^{237} \mathrm{~Np}$, and lower priority for ${ }^{238,239} \mathrm{~Np}$. While absolute branching ratios are required in most instances, for NDA applications, intensities are often utilized, and these can be determined at a much higher accuracy than absolute values. A more specific analysis of needs relating to NDA and forensics will be supplied to NA-22 directly, as these could not be openly discussed.

The consensus was that significant progress has been made with decay data for reactor antineutrinos and decay heat. Through various interagency funding opportunities involving NP, ND, and NA-22, approximately 100 fission fragments have been (or will soon be) studied using the technique of total absorption gamma-ray spectroscopy (TAGS). These measurements will make tremendous advances in our ability to reliably predict the antineutrino spectrum from a nuclear reactor. Since these studies addressed all isotopes considered high-priority, there is no pressing need for additional TAGS measurements at this time. TAGS measurements allow for extraction of beta-feeding intensities. From this one can determine the beta spectrum. Approximately 25\% of the fragments decay by first forbidden transitions and require theoretical corrections to the shape of their spectra. Currently, these so-called shape factors have not been measured in the fission fragment region, so measurements of the shape of the beta spectrum are required to complement the TAGS measurements and to determine the magnitude of this correction term.

An overarching topic which arose throughout the discussion was the need for better tools to interact with decay databases. ENSDF is a non-numeric, 80-column American Standard Code for Information Interchange (ASCI) format. It is heavily embedded with comments, so parsing the database is quite difficult. There was widespread consensus among the participants regarding the need for an API package to allow users to quickly and easily parse the database for user-defined observables. A few participants mentioned that they had developed their own ad-hoc parser tuned to their specific needs. An interactive mechanism using a platform such as GitHub in which people can contribute code and/or provide suggestions was recommended to allow for community-based improvements. There was also discussion on ways to supplement the ENSDF database. The uncertainties in ENSDF can be numeric, non-numeric $(\sim,<,>)$ or non-existent; this makes interpreting ENSDF with a program very problematic. A more consistent treatment of uncertainties was desired. In addition, there were requests to incorporate a flag into ENSDF to indicate data quality.

\section{A.4 Actinide Cross Sections}

\section{Session Leaders: Susan Hogle, ORNL and Lee Bernstein, LBL/UCB}

This session was focused on supporting forensics and reactor calculations by solving the network of neutron absorption, decay, and production within the actinide network (see Figure A-2) with a focus on the minor actinides. Many of the minor actinides of interest have short half-lives, so they are difficult to measure, which leads to large uncertainties in their cross sections. However, these cross sections are important for understanding the production of measurable quantities when actinides are exposed to a large neutron flux, and small uncertainties can compound quickly, leading to large uncertainties in accumulation and decay rates. Changes to one data-point can break previously established data points within the complex, interconnected web. The goal of this session was to determine how to improve the quality of this overall actinide network through a combination of measurement and theory improvements, identifying which measurements should and could be made, what resources are needed to make them, and what alternative techniques can be applied to fill in immeasurable gaps. 


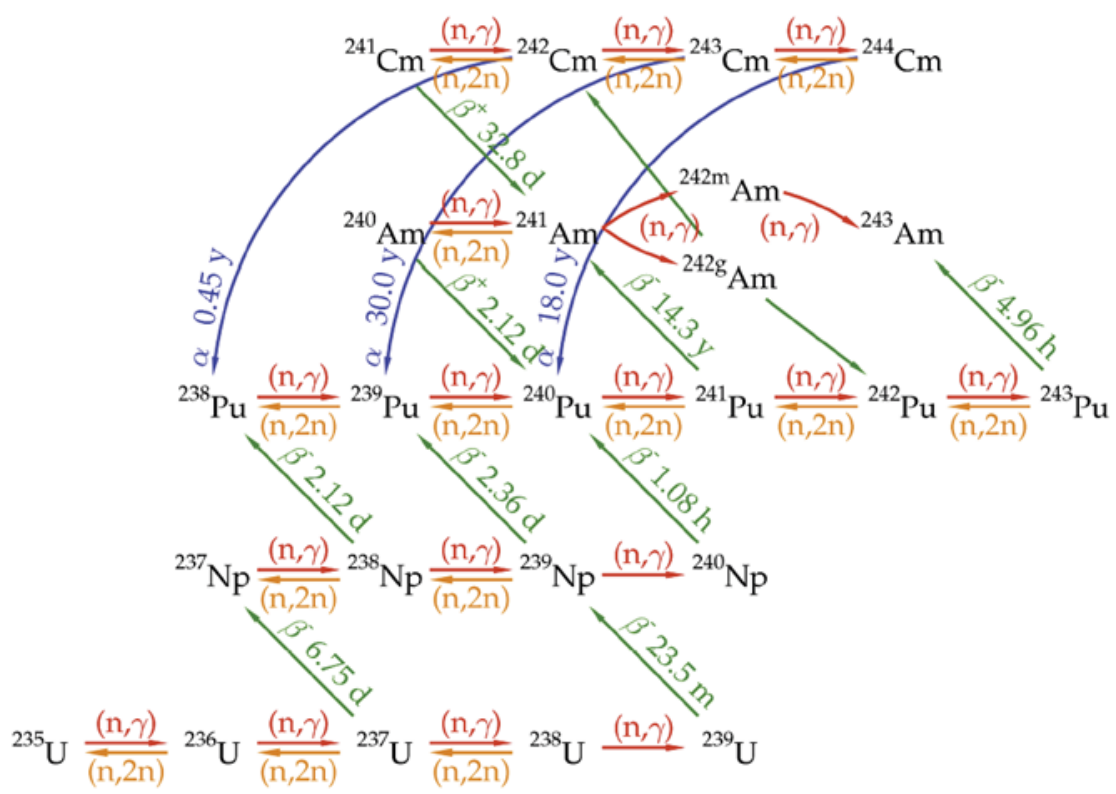

Figure A-2. The actinide network of capture and decay.

\section{A.4.1 Current state of the data}

The isotopes that have seen the greatest study are ${ }^{235} \mathrm{U},{ }^{238} \mathrm{U}$, and ${ }^{239} \mathrm{Pu}$. Most work surrounding actinide cross sections has been performed in the context of fission reactors or nuclear forensics. However, data performance varies, depending on the specific application. Many compensating errors could be present, leading to undiscovered uncertainties. DNN R\&D work has largely focused on U and Pu isotopes, with a smaller body of work focused on Np and Am. Recent evaluations at LLNL include ${ }^{236,237,238} \mathrm{Pu}$, as well as Am isotopes. Historically, data collection has not always included evaluations, or it may have included data evaluation only for application specific uses; these data may not have been made available to all users.

The FIRE collaboration is examining advanced models of fission and incorporating the rapid neutron capture process formation of heavy elements into models. This project - a collaboration with LLNL/LANL, Notre Dame, and NCSU - is a successful, mutually beneficial collaboration supported by NA-22 and Office of Science. However, the deliverable resulting from this project will likely not directly provide fission data to the user, it will provide theoretical input to fission yield evaluation efforts.

The IAEA CIELO project has created new evaluations of ${ }^{235} \mathrm{U},{ }^{238} \mathrm{U}$, and ${ }^{239} \mathrm{Pu}$, and in the next cycle, ${ }^{240} \mathrm{Pu}$ and ${ }^{242} \mathrm{Pu}$ will be examined. Recent TUNL measurements have included ${ }^{238} \mathrm{U}(\mathrm{n}, 2 \mathrm{n})$ and $(\mathrm{n}, \mathrm{g})$ (0.1-15 MeV), and Chi-Nu has measured scattering on ${ }^{235} \mathrm{U}$ and ${ }^{239} \mathrm{Pu}$.

There are many important reaction types for the actinides, and there is significant overlap between this and other workshop sessions. Much of the discussion focused on $(\mathrm{n}, \gamma)$, but for many of these same isotopes, fission, decay, inelastic scattering, $(n, 2 n)$, and $(n, x n)$ are also important.

\section{A.4.2 Prioritized needs}

\section{A.4.2.1 Isotopes of Interest}

1. $\mathrm{U}$ 
a. ${ }^{239} \mathrm{U}(\mathrm{n}, \mathrm{g})$ and $(\mathrm{n}, \mathrm{f})$ in lower energy regions

b. ${ }^{237} \mathrm{U}$

c. ${ }^{235} \mathrm{U}$, esp. for $100 \mathrm{eV}$ to $0.5 \mathrm{MeV}$ or $1 \mathrm{MeV}$

2. $\mathrm{Np}$

a. ${ }^{237} \mathrm{~Np},{ }^{238} \mathrm{~Np}$ (models not matching observations for either)

3. $\mathrm{Pu}$

a. Metastable forms

b. ${ }^{238} \mathrm{Pu}$ discrepancies at higher energies

c. ${ }^{239} \mathrm{Pu}$, esp. for $100 \mathrm{eV}$ to $0.5 \mathrm{MeV}$ or $1 \mathrm{MeV}$

d. ${ }^{236} \mathrm{Pu}$

4. Am

a. ${ }^{243} \mathrm{Am}$ (drives curium production)

b. ${ }^{241} \mathrm{Am}$ (state of data is better than that of ${ }^{243} \mathrm{Am}$ )

5. $\mathrm{Cm}$

a. ${ }^{244} \mathrm{Cm}$ capture and fission in thermal and fast reactor spectrum

b. ${ }^{244} \mathrm{Cm} \mathrm{P}(\mathrm{nu})$ (overlap with fission needs)

\section{A.4.2.2 Structure-Informed Reaction Modeling Needs}

Development of theory and modeling is necessary to help fill in gaps in data needs that cannot be met through traditional measurements due to unavailability of material, difficulty of material isolation or targets preparation, short half-lives, etc. Better understanding of energy densities and other parameters improves the usefulness of experiments by enabling better interpretation of results and investigation when data do not match up. Models currently exist, but with little experimental backing and with many unverified assumptions. While fission modeling has experienced recent improvements, (n,g) and (n,2n) have been poorly represented. Because the deliverables of this work are less tangible than direct measurements, it has been difficult to justify their impact to the user.

Parameters of interest for structure-informed reaction modeling include:
a. Discrete structure
b. Radiative strength / strength functions
c. Level / energy densities
d. Excited states
e. Evaporation spectra
f. Average spin

\section{A.4.2.3 Facility and Target Needs}

There is a need for monoenergetic neutrons with high flux $\left(>10^{12} \mathrm{n} / \mathrm{cm}^{2}-\mathrm{s}\right)$. Currently, Ohio can meet this need up to $\sim 500 \mathrm{keV}$. The lead spectrometer has been useful for lower energy measurements and has been applied to Cm isotopes, as well as ${ }^{238} \mathrm{~Np}$ and other minor actinides. The lead spectrometer can measure fission cross sections using a target consisting of less than $20 \mu \mathrm{g}$ of material.

\section{A.4.2.4 Evaluation and Uncertainty Needs}

There is a known problem with new cross section experimental data accumulating and not being evaluated or incorporated into ENDF. Evaluation of existing data and dissemination to ENDF, or at least to internal databases, is important. Publication in peer-reviewed journal articles and incorporation in EXFOR should be required for all projects, and data sharing should be required as part of the data management plan. 
Projects often run out of funding, or funding is not requested to perform the evaluation. Evaluation requires coordination between experts at all stages of the project, and funding for an evaluator should be included in the initial proposal. Program managers should look for inclusion of the evaluation in a project proposal and score the proposal more favorably. Similar problems occur with validation and dissemination of data, which may take years to complete after a project deliverable has been met. In general, experimental, theory, and data processing communities should be in open communication throughout the data development process.

It is important to include uncertainty quantification for actinide cross sections. Much of these data are not bounded at all, particularly data for surrogate reactions in which theory is heavily relied upon and there is no validation of the data. Uncertainty values should at the very least be bounded, as even a $20 \%$ uncertainty is a large improvement over having no data. Uncertainty has been better quantified to date for thermal energies.

\section{A.4.3 Recommended actions}

A global data collection methodology should be established that will ultimately apply to many isotopes. Initially, this methodology should be applied to an important isotope that can be easily measured to demonstrate good procedure, starting with theory and experiments, all the way through implementation. It will likely be necessary to package this methodology with tangible measurement deliverables, thus improving its chances of being funded. Collaboration and joint proposals are encouraged to bring together many different experts across laboratory lines. Incorporating experts into experimental, theory, and data processing areas will assure that quality work is performed and disseminated.

Integral validation data from reactor irradiations should be explored for cases in which conditions can be properly characterized.

\section{A.5 Gamma-Induced Reactions}

\section{Session Leader: Brian Quiter, LBNL}

The primary driver for assessing the status of photon-induced nuclear data was a request from DNDO in which it was conveyed that simulations resulted in predictions of "signatures" from photofission that were induced within cargo inspection scenarios. The signatures were ten times larger than those that had been experimentally observed. Since active interrogation with photons is of interest for nonproliferation missions, and since DNN R\&D is funding development of a fieldable quasi-monoenergetic photon source, it was determined that nuclear data needs for active interrogation should be addressed. In addition to this primary charge, session participants discussed other needs and applications of photon-induced reactions and data quality. Overall, the reactions and phenomena discussed include the following:

- Bremsstrahlung production cross sections and modeling

- Photonuclear reaction cross sections and implementation in modeling codes

o Photofission: cross sections and emitted particle multiplicities and distributions

o Other photonuclear reactions

- More complex photon behavior

o Polarized photons and photon beams with orbital angular momentum

- Cross sections and secondary particle behavior

o Gamma mirroring behavior

- Photon attenuation behavior

0 At and near $x$ ray K-edge energies.

o At multiple $\mathrm{MeVs}$ 
- Nuclear resonance fluorescence phenomena

- Tabulations of shielding and detector response behavior to facilitate/accelerate applications modeling

\section{A.5.1 Data assessment for photofission model-simulation comparison}

Cargo inspection is subject to an energy limit of $10 \mathrm{MeV}$ because there are concerns about inducing photonuclear reactions and activating materials. The session participants discussed the quality of nuclear data as they relate to all the phenomena required to simulate photofission cargo inspection. This includes bremsstrahlung production data, $(\gamma, \mathrm{f})$ and $(\gamma, \mathrm{xn})$ data, and the nuclear data pipeline.

The participants agreed that while bremsstrahlung-integrated cross sections are well understood (error $<5 \%$ ), the relative production rates near the beam endpoint energy could be incorrect by up to $50 \%$. Literature on the subject also indicates that near endpoint energy behavior may not be well defined by the theoretical tabulations used in Monte Carlo codes [1]. Similarly, differential photonuclear cross sections are generally well understood, and the dominant feature tends to be the giant dipole resonance (GDR), particularly in energy regimes $<10 \mathrm{MeV}$. The participants indicated that interaction cross sections are likely understood to $>7 \%$ in the GDR region, but in the low-energy tail portion of the GDR (threshold to a few $\mathrm{MeV}$ above threshold), errors of $20 \%$ or more are probable. When these errors are convolved with bremsstrahlung production rate uncertainties, session participants observed that errors of about a factor of two could be reasonable. However, this remained well below the factor of 10 reported by DNDO.

Given that reported differences between simulation and measurement were still substantially larger than could be explained by interaction cross sections alone, two additional sources of error were hypothesized: inaccurate secondary particle production rates and missing physics within the MCNP simulation. Both sources of error were considered probable, depending on the exact sort of phenomenon that was being modeled and measured; missing or inaccurate $(\gamma, \mathrm{xn})$ cross sections will result in much lower backgrounds than predicted. It is uncertain whether the particle production phenomena were not simulated due to missing data or due to a failure to include existing data in the simulation. The assembled session participants concluded that both photo-induced secondary particle production rates are generally insufficiently known and that the nuclear data pipeline could be improved.

Photofission daughter behavior was thought to be well understood, and the photo-fission production rates into the 6 beta-delayed groups have recently been improved, but otherwise, energy dependence of fission yields, angular distributions, and neutron-gamma correlations are essentially considered unknown.

\section{A.5.2 Other photonuclear data applications}

Subcritical assembly experiments may want to include photo-induced neutron sources in their models. This would be an important DNN application that would require significant improvements to $(\gamma, \alpha)$ and $(\gamma, \mathrm{xn})$ cross sections. Photonuclear reaction cross section databases that are used to describe isotope production were described as nearly empty, and the medical isotopes community may have shared interests in measuring some cross sections and/or developing shared methods. Presently the European TALYS code and resulting TENDL libraries [2] are undergoing some benchmarking activities in support of these applications.

Nondestructive assay (NDA) methods using XRF and NRF are relevant applications for safeguards, specifically for HKED measurements used for input accountability in reprocessing facilities. There is a need for more precise data on the magnitude and energy-dependent shape of attenuation coefficients near K edge energies. Since attenuation measurements can occur over many path-lengths, small data errors propagate multiplicatively. This may also be an issue at photon energies used for radiography. The cross 
sections for the strongest NRF transition are sufficiently well known $(<20 \%)$ to perform modeling for major actinides, but the data do not exist or are not tabulated in evaluation presently to allow sufficiently accurate modeling integral measurements, or relevant elastic scatter that results in NRF backgrounds. Both facts hinder evaluation of NRF as a fieldable technology, and the latter fact causes many studies that attempt to predict applicability of NRF to be overly optimistic.

Additionally, new photon sources are being brought online, and new photon physics phenomena continue to be studied. Laser-Compton sources enable beams with narrower energy spreads than bremsstrahlung and are being developed by DNN with the intent of being used in future applications. These sources can produce polarized photon beams, and they may be able to produce beams with orbital angular momentum. The study of polarized beam nuclear interactions has been conducted, and phenomena that may be relevant for DNN exist, but they cannot be predicted by MCNP, nor are they tabulated in ENDF. Further study of the usefulness of polarized beam interactions is required.

Photon-induced reaction and secondary production cross section errors and omissions also cause uncertainty and lack of confidence in photon-induced dose rate calculations. This may contribute to arbitrary limits (e.g., $10 \mathrm{MeV}$ cargo interrogation limit) that affect mission-relevant capabilities, may confound survivability determinations, and may cause other unanticipated impacts on DOE missions.

Other difficult-to-simulate photon phenomena could also benefit applications studies if there were tabulations. These phenomena include collimator and shielding effects and detector responses akin to GADRAS tabulations and dose build-up tables.

Generally, given the poor the state of nuclear data with respect to photon-induced reactions, direct experiments to measure phenomena are preferred to attempt to predict rates through Monte Carlo.

\section{A.5.3 Needs prioritization}

The top two nuclear data needs were determined by group consensus, while the others had no clear prioritization.

1. Determine the extent and cause of inability to accurately model photofission in cargo scanning settings

2. Improve data for secondary particle multiplicities and energy distributions

3. Add more photon physics to nuclear data tabulations and codes

4. Develop detailed attenuation coefficients for XRF and radiography

5. Improve accuracy of bremsstrahlung spectrum characterization between 1 and $10 \mathrm{MeV}$

6. Tabulate collective photon interaction phenomena

7. Study the unclear status of Cherenkov light generation data for photon detectors (understood much better for heavy charged particles)

\section{A.5.4 Recommended Actions}

1. Either:

a. Conduct small-scale demonstration experiment to obtain photofission signal data in a relevant operational scenario and compare with MCPN6 simulations (less expensive, lower impact);

b. Conduct well-controlled, simple-to-model cross section measurements at a facility such as HIGS to measure the relevant nuclear data for photo-fission cargo interrogation (most costly but broader impact); or

c. Piggy-back on other fission data collections: transfer neutron-induced fission cross section measurement setup to HIGS to perform similar measurements with photons (reduce cost of $b$ while maintaining impact). 
2. Perform data evaluation to ensure that new photonuclear data are reaching MCNP

3. Team with European and medical physics communities to possibly establish methods to measure secondary particle production cross sections.

\section{A.5.5 Bibliography}

[1] D. H. Rester and W. E. Dance, Phys. Rev. 161, 85 (1967).

[2] http://www.talys.eu/

\section{A.6 Neutron Capture and Associated Spectra, and Inelastic Neutron Scattering and Associated Spectra (combined summary)}

\section{Session Leader: Lee Bernstein, LBNL/UCB}

NDREW Session 1B (Capture Gamma and Associated Spectra) and 2B (Inelastic Scatter and Associated Spectra) were narrowly focused on addressing $\gamma$-ray spectral nuclear data needs following $(n, \gamma)$ and $\left(n, n^{\prime} \gamma\right)$ to support active interrogation methods for nonproliferation missions. This concentration session was chosen based on discussions in the last two Nuclear Data Working Group (NDWG) meetings (BNL/CSEWG 11/17 and LANL 12/17) since it was thought that the case for the importance of (n,n' $\gamma$ ) for neutron transport was already established during the past year's NDWG process, culminating in the Nuclear Data Exchange Meeting on 4/14/16. Similarly, the narrow focus for the NDREW (n, $\gamma$ ) session was implemented in part to avoid overlap with other sessions at NDREW, which focused on the production/destruction aspects of neutron capture on actinides for forensics. Neutron capture on nonactinides has been an ongoing effort of the Nuclear Criticality Safety Program (NCSP), focusing primarily on structural materials and those used in nuclear materials processing.

The attendees concurred that the gamma-ray spectral information in ENDF needs significant improvement for the proper modeling of active and passive neutron interrogation data. This was particularly the case for the continuum component of the spectrum, which accounts for most of the spectrum following neutron capture and remains largely ignored. Whether this component should reside in ENDF (since it is the most important for reactions) or ENSDF (since it concerns levels below the neutron separation energy) remained open for discussion. It was clear that any effort to improve this data would overlap with the currently running IAEA Coordinated Research Project on Radiative Strength Functions.

One approach to improving $\gamma$-ray spectral data in ENDF was to optimize the modeling of existing $(\mathrm{n}, \gamma)$ and (n,n' $\gamma$ ) data using a reaction modeling code (EMPIRE, Talys, CoH3 etc.) and then incorporate modeled spectra into the database. This approach has the benefit of following the same methodology used for cross section evaluations. However, it does not allow for the fact that the $\gamma$-ray spectrum and multiplicity differ from event to event, making this sort of data more like fission data, which produce a range of products and neutrons in a stochastic manner. A more useful approach, particularly for deterministic transport modeling, would be to use a $\gamma$-ray event generator along the lines of the FREYA package for use in fission. The CGM package developed at LANL might serve this need if it were tuned to reproduce existing data. Furthermore, it was noted that not only evaluation, but also appropriate data processing, is needed to address the unusual aspects of $\gamma$-ray spectral data.

Both approaches require good, measured $\gamma$-ray spectra data; data would preferably include both discrete and continuous components. At thermal energies, the discrete peak data contained in the Evaluated Gamma Activation File (EGAF) appear ready to be incorporated into ENDF (or GND, once it is released) at a modest effort level. However, there was clearly a need for additional measurements of $(n, \gamma)$ at higher 
( $\mathrm{keV}$ to $\mathrm{MeV}$ ) energies to support active interrogations with DD and DT neutron sources. The DANCE array at LANL, CERN-NTOF, and the GAINS spectrometer at GELINA are currently producing data along these lines. However, these data are focused on specific nuclides, such as the Big 3 actinides, and only up to a few hundred keV neutron energy.

In addition to these laboratories that are producing ( $\mathrm{n}, \mathrm{x} \gamma$ ), there are significant smaller, university-based facilities with unique capabilities to address these needs. Some of these are particularly well-suited to address $\gamma$-ray spectral data needs due to their unique combination of spectrometry (e.g., swinger magnets, long TOF-lines, etc...) and an absence of extremely high-energy neutrons that can contaminate data due to multiple scattering. A list of these facilities includes RPI, Ohio University, Kentucky, and most recently, LBNL/UCB. Participants recommended that a list of domestic and international facilities that can address these needs be compiled and periodically updated.

Obtaining clean capture and inelastic scattering $\gamma$-ray spectra on the Big 3 actinides poses an additional problem. Virtually all measurements of $(n, \gamma)$ and $\left(n, n^{\prime} \gamma\right)$ on these nuclides are contaminated by have a strong (n,f $\gamma$ ) prompt signal. This means that the evaluator must separate these components based solely on uncalibrated modeling. The only data not subject to this issue would be fission-tagged thin target data, of which very little exist. It is possible that triply coincident data from direct reactions such as U/Pu(d,pf $\gamma$ ), where the outgoing proton and fission fragment are detected in coincidence with the $\gamma$-rays, could be used to aid in this process.

At higher energies, (n,n' $\gamma$ ) becomes increasingly important relative to $(n, \gamma)$, and the quality and quantity of the data decrease significantly. There is a body of heritage data from a team of researchers working at the University of Kentucky, RPI, and a number of facilities in Europe, but much of this was taken for nuclear structure studies and would require additional analysis. This would almost certainly be conducted by the experimental team that produced it and would be converted into useful cross sections. Overall, there is a lack of measured data. The only significant standing body of (n,n' $\gamma$ ) is the Atlas of Gamma-ray Spectra from the Scattering of Reactor Fast Neutrons, also known as the Baghdad Atlas. This was developed for a broad fast reactor spectrum that is not likely to be representative of DD and DT neutron sources.

Several other needs were clearly articulated by the group. One was the need for a sensitivity tool along the lines of TSUNAMI that could be used to determine where new measurements or evaluations are most needed. There was also consensus that at least one benchmarks facility with well-defined neutron spectra (e.g., DD, DT, PFNS) would be invaluable for testing and validating $\gamma$-ray spectral data. Another issue that was observed was that virtually all data lacked angular distribution information, which may or may not be shown to be useful for active interrogation measurements.

Lastly, attendees also noted that much of the physics input for modeling $(n, \gamma)$ needed to address DNN data needs is similar to the data needed by the nuclear astrophysics community, since virtually all heavy nuclei are formed via neutron capture. There is also significant overlap with stewardship science to model archival radiochemical data from nuclear testing and from nuclear energy to model the burnup of radionuclides in spent fuel in NDA measurements.

In both sessions, a prioritized list of recommended activities to be pursued to address deficiencies in $(\mathrm{n}, \mathrm{x} \gamma)$ spectral data was agreed upon. These are reprised below. 


\section{A.6.1 Prioritized Plan of Action - Capture Gammas}

1. The initial focus area should be on improvement in the evaluation and post-processing of existing experimental data for capture gamma-ray spectra.

a) Targeted measurements above $E_{n}>25 \mathrm{keV}$ (Data goes from sparse to 0 as $E_{n}$ goes up)

b) A continuously updated inventory of experimental capabilities would help guide these measurements.

c) We need data, in units of mb or per capture reaction, including quasi-continuum and discrete data, if the capture signal is significant.

d) The quality of data needed depends on the details of the application.

2. A benchmark for a specific application_(e.g., prompt- $\gamma$ from DD neutrons) would help define the required sensitivity.

3. We should consider improving/expanding the methodology_used to put data in the database

a) Creation of data sets_for inclusion into ENDF using sophisticated modeling

b) Use of a gamma-ray cascade event generator (with correlations) for incorporation into or use with transport codes. (CGM and other tools exist that might fill this need)

4. A continued program of $(n, \gamma)$ activation is needed to develop and maintain capabilities and expertise. NCSP is addressing this area (often with single points of failure)

\section{A.6.2 Prioritized Plan of Action - Inelastic Scattering}

1. Develop benchmarks for relevant applications (e.g., prompt- $\gamma$ from DD, DT, PFNS) to define the approach and its required sensitivity.

a) Remember to include (n,n') to isomers for post-det forensics (for nonactinides).

b) The detection system response must be well-characterized over the entire range of measurement.

2. Convert existing data into cross sections, and generate new mission-relevant data, including the nuclear data pipeline*

a) A continuously updated inventory of experimental capabilities would help guide measurements.

b) Data are needed in units of mb or per scatter reaction, including quasi-continuum and discrete data, and angular correlation if the signal is significant.

3. A tool to determine sensitivity for a given system is needed for both application and benchmark analysis.

a) Develop eigenvalue-like $\mathrm{S} / \mathrm{U}$ tools for gamma-ray and neutron spectral data.

b) Subject matter experts are still essential to interpreting the output of this tool.

4. We should consider improving/expanding the current methodology for data inclusion into ENDF

a) One approach is to optimize reaction model output relative to measured data.

b) Another approach, appropriate for nuclei with fluctuations, is to put measurements directly into the model. Improvements are needed in the inputs to the reaction model (level density, pre-equilibrium, general structural inputs). This is a cross-cutting contribution to other missions.

\section{A.7 (Alpha,n) reactions}

\section{Session Lead: Matt Devlin, LANL}

This section focused on the nuclear data required for use of $(\alpha, n)$ reactions for nonproliferation applications. Participants discussed (1) current and past work at the Naval Nuclear Lab, which identified issues in the outgoing neutron spectrum, (2) previously funded differential measurements of ${ }^{17,18} \mathrm{O}(\alpha, \mathrm{n})$ 
and ${ }^{19} \mathrm{~F}(\alpha, \mathrm{n})$ using alpha beams and inverse kinematics, and (3) evaluation methods that provide covariances using an R-Matrix formalism for these reactions.

Since the nuclear data needs for nonproliferation applications primarily concern the big three isotopes in oxide and fluoride compounds $\left({ }^{17,18} \mathrm{O}\right.$ and $\left.{ }^{19} \mathrm{~F}\right)$, it was noted that previously funded work had begun addressing the differential cross sections. Nonetheless, there are still deficiencies in the energy range and resolution of differential measurements. For oxygen, fluorine, and other elements, differential data are needed over the energy range of $<1 \mathrm{MeV}$ to $9 \mathrm{MeV}$ with consistency and $2 \%$ resolution. Data taken by the ORNL collaboration for ${ }^{19} \mathrm{~F}(\alpha, \mathrm{n})$ from $6.7 \mathrm{MeV}$ to $8 \mathrm{MeV}$ were not analyzed due to funding limitations. Some participants mentioned that the measured but unanalyzed neutron angular distribution data would also improve neutron transport simulations.

Many of the differential measurements in the literature use carbon $(\alpha, n)$ as a reference reaction, and improved carbon data are important. A lower priority is the measurement of $(\alpha, n)$ reactions on many of the nonproliferation-relevant, stable, light elements such as structural materials and those mixed with alpha emitters. For evaluations, theoretical calculations and improvements are needed in the R matrix treatment of $(\alpha, n)$ reactions to extend the evaluations to higher energy and to explicitly include threebody exit channels. Participants considered new evaluations to be a low-cost, high-impact program that would directly impact the need for improved uncertainty quantification.

For integral data needs, requests were made for (1) improved benchmarks using well-calibrated sources for validation, (2) for thick target measurements needed for validation of the differential data and simulations using these data, and (3) for improvements in methods, calculations, and simulations. Of particular interest were improvements to SOURCES4C, with suggestions ranging from added information, active maintenance of the evaluations and format, and outright replacement with a more modern, versatile code.

Participants discussed measuring associated $\gamma$-ray data with new measurements, measuring accurate outgoing neutron energy spectra, improving the evaluations of existing data, and improving the knowledge of stopping powers and energy loss in mixed materials. Matrix effects cause variations in neutron rates from different material forms and have been noted experimentally, but this issue is poorly understood and might be a fertile area of study.

\section{A.8 Development of Benchmark Exercises}

\section{Session Leads: Rian Bahran, LANL and Sean Stave, NNSA, Safeguards Technology}

Benchmarks are well controlled experiments of a relevant system used to compare calculated values to experimental values. This comparison using sensitivity and uncertainty tools can shed light on the most

important sources of uncertainty in the system. Benchmarks specifically relate to nuclear data in that they can validate the data used in models. The relationship between benchmarks (validation experiments), differential and integral nuclear data, and models is shown in Figure A-3 below. For nonproliferation, benchmarks can provide information used to prioritize research.

Participants in the benchmarking session discussed existing benchmarks and methods used for criticality safety and those used for safeguards. Benchmarks for nuclear data can take the form of integral measurements, quasi-integral measurements, and validation experiments. 


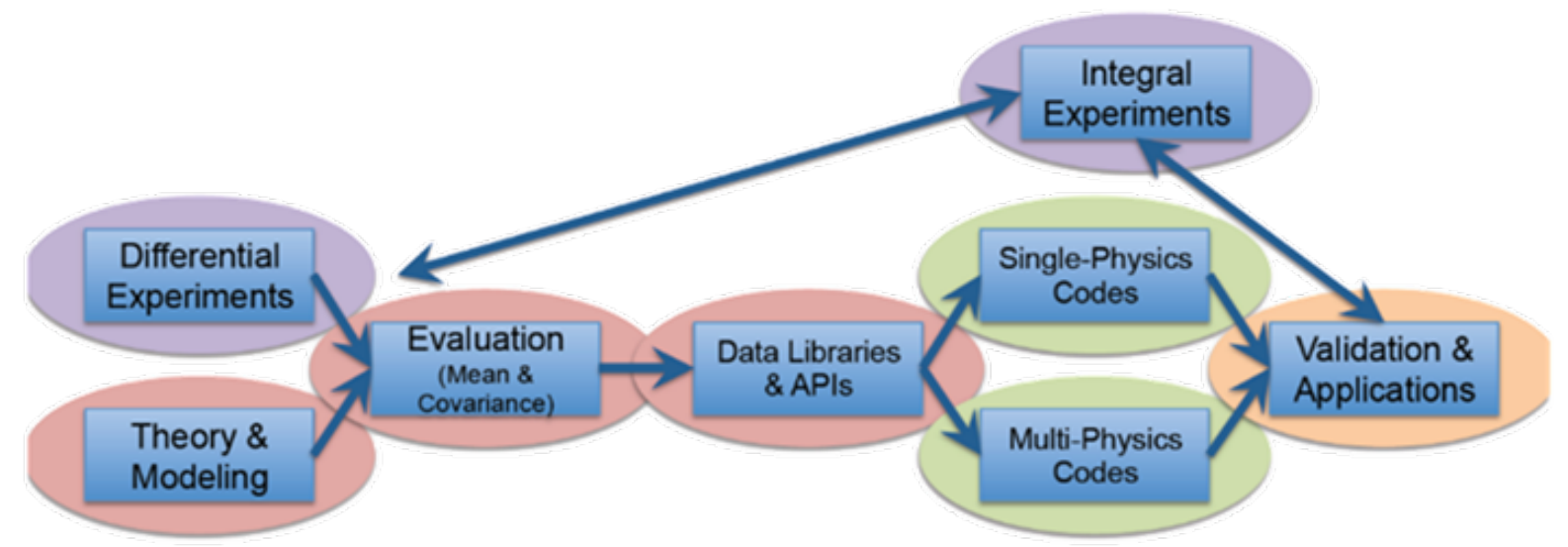

Figure A-3. Flow of information to validate nuclear data libraries using integral experiments.

The criticality safety and reactor community have formalized methods to compare models to benchmark experiments. An example that has been used for safeguards is the determination of the sources of uncertainty in decay heat calculations for spent fuel assemblies. Figure A-4 shows the results of an uncertainty study of the impact of operating data, fuel design data, fission yield data, and cross section data on the total calculated uncertainty when compared to measured decay heat. In this case, the models used have been extensively validated, and most uncertainty comes from the input parameters. The study's results determined that the largest uncertainty in decay heat calculations comes from the reactor operation parameters and the cross sections and that the uncertainty increases for high burnup fuel and long cooling times. This analysis determined that more work is required in cross section data to enable the use of gamma spectral measurements and simulations as a replacement for decay heat measurements of spent fuel assemblies. These types of studies are useful, but only if they are well documented and read by those who use them.

The Passive Nondestructive Assay (PANDA) manual, is a guide for various safeguards measurements with data provided to be used to estimate values such as mass values of measured items. These data should be updated, but they are based on specific benchmark experiments with designated detector systems. Since many safeguards measurements rely on well-calibrated, well-characterized systems that have been in use for many years, experiments using these systems can be incorporated into a benchmark database and uncertainty analysis. 

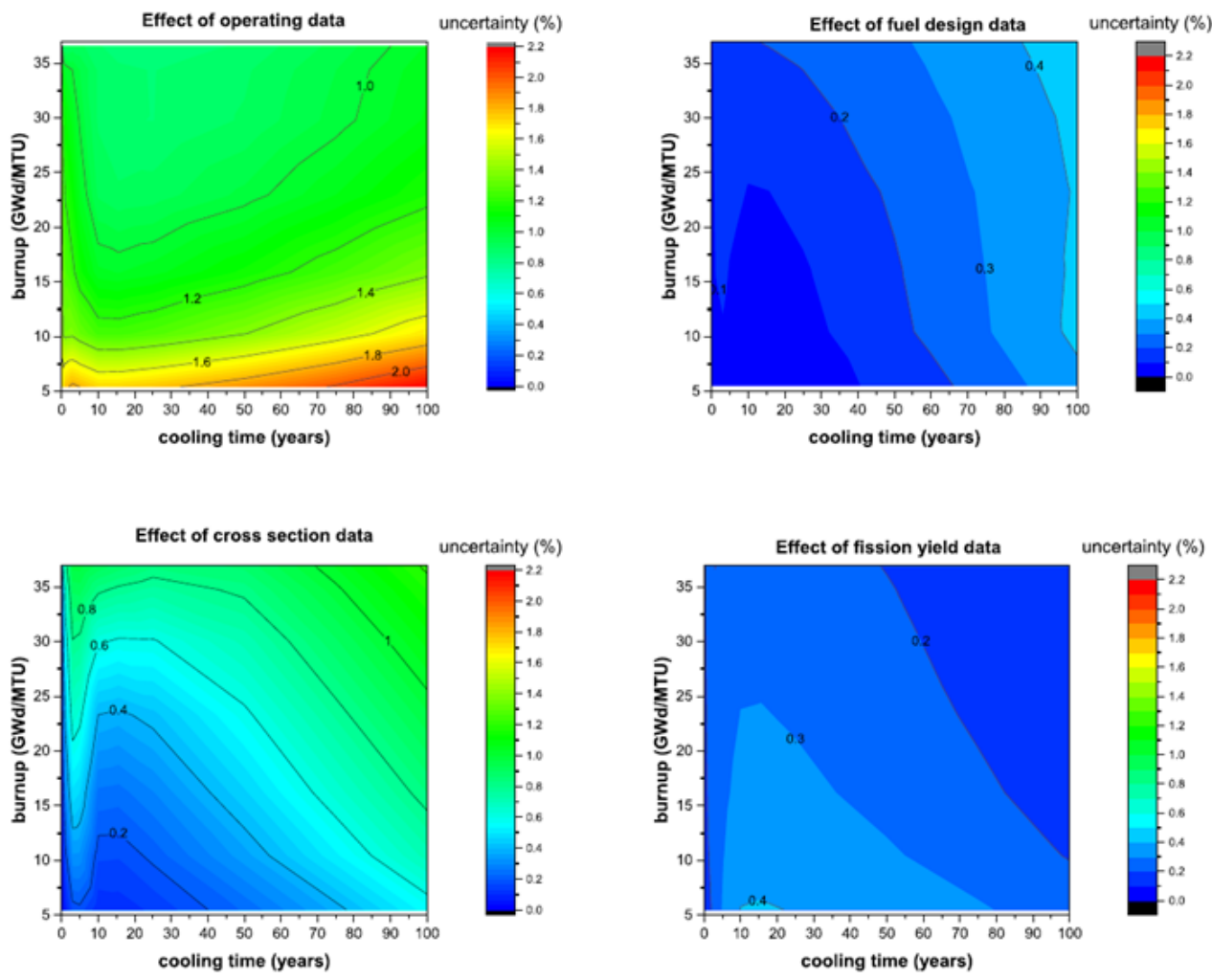

Figure A-4. Decay heat uncertainty as a function of burnup and cooling time.

\section{A.8.1 Recommendations:}

- Create a dedicated suite of nonproliferation benchmarks and validation sets: the Nonproliferation Benchmark and Validation Suite Repository.

- Develop capabilities for uncertainties, sensitivities, documentation, accessibility, peer-review, and a data management plan.

- Ensure that a significant investment is made in the benchmark repository to ensure that it makes a measurable impact.

- Ensure that additional development in sensitivity and uncertainty methods, as well as detector response model development, are implemented to support the effort.

- Use the Nuclear Data Working Group model to plan the effort effectively.

Overall, the workshop discussion was the first of it's kind to determine the requirements for benchmark experiments not only to determine nuclear data uncertainties, but also to determine uncertainty contributions from all input parameters, system models, and measurement uncertainties. Further discussion is required to determine how to move forward, and a working group consisting of multiple disciplines and expertise would be a good way to begin. Benchmark experiments and the management of related data can be expensive, so it is important to determine first what data are already available to be used as a benchmark and how to collect and analyze that data. In addition, a list should be created of wellcharacterized measurement systems and uncertainty/sensitivity tools that are ready and available to be used for benchmarks.

The uncertainty/sensitivity analysis based on benchmarks should have the goal of gaining understanding of the quantity and source of uncertainty in real-world measurements for nonproliferation. The study must result in actionable data to guide future research funding that will have a positive impact on the mission. 


\section{A.9 Uncertainty, Sensitivity and Covariance}

\section{Session Lead: Bradley T. Rearden, ORNL}

The application of sensitivity analysis, covariance data, and uncertainty quantification continue to emerge as preferred techniques for identifying the input parameters and data that are most important for a given calculation, how confidence in reported nuclear data values influence confidence in the output results, and how uncertainties in system specifications influence the precision of output results.

\section{A.9.1 Analytical methods}

There are two general classes of approach in uncertainty quantification: stochastic sampling and sensitivity methods. In stochastic sampling, many trials of the full calculation are repeated where input data are varied within their given uncertainties, accounting for any known correlations in these input values. The distributions of the output quantities resulting from many calculations with varying input quantify the uncertainty in the computed quantities. Stochastic sampling enables virtually any uncertain quantity to be sampled, including technological parameters such as material compositions and dimensions; values predicted by other means and provided as input to the present calculation, such as source strength and temperature; and nuclear data where the uncertainties in many types of data can be simultaneously sampled, including reaction cross sections, fission product yields, decay constants, branching ratios, and secondary particle emissions. Generally, the sampling process occurs outside of the computational code, and perturbed inputs are provided to the standard code, whether in the form of updated input files or updated nuclear data libraries. A driver that encompasses the calculation performs the data sampling and reports the uncertainties in the output quantities of interest, which can be generated by a single code or a series of codes with data transferred between them. The stochastic sampling approach is very useful in simultaneously determining uncertainties in many output quantities due to many input quantities and has been successfully applied to complex analyses, including the determination of the uncertainty in hundreds of isotopic compositions in irradiated nuclear fuel due to millions of uncertain nuclear data parameters. However, due to the statistical nature of this approach, it is difficult to determine the impact of any given uncertain quantity on the uncertainty of an output result.

Sensitivity analysis provides precise data regarding the impact of any input quantity (e.g., a single reaction cross section for a single nuclide at a given energy point) on the output data (e.g., neutron spectrum). Sensitivity analysis clearly identifies which inputs most influence the output. A sensitivity coefficient represents the percent change in an output parameter due to a percent change in an input parameter of interest. For reaction cross sections, these results are often shown as sensitivity profiles, where the expected change in the output value to the energy-dependent cross section across the entire energy range for a given nuclide and reaction are displayed. The uncertainties in each of the input values are propagated through these sensitivity coefficients to an uncertainty in the output result, not only as an integral of all sources of uncertainty, but also with the availability of individual sources of uncertainty. The sensitivity approach provides insights into the input data that most influence the output results and have broad application in system design optimization and validation. The generation of sensitivity coefficients requires direct enhancement of the solution algorithms within the physics codes to provide not only the computed quantity of interest, but also the sensitivity of that quantity to input data. This is generally based on the first-order derivative of the output result with respect to the input data. Where the derivatives are imbedded in the code, the sensitivity of an output quantity to all input data is simultaneously generated in fine detail. A challenge with these methods is that derivatives must be available within an individual physics module, generally through adjoint functions, importance functions, or through differentiation and chain rule propagation of the mathematical model within the code. Where different codes are employed through the calculation—such as in depletion, where sensitivities in the 
neutron spectrum impact sensitivities in fission product yields—a chaining approach must be applied to propagate sensitivity from one code to the next.

\section{Uncertainty Quantification Tools}

\begin{tabular}{|l|l|}
\hline Tool & Capabilities \\
\hline Kiwi (LLNL) & $\begin{array}{l}\text { Create perturbed ACE libraries for MCNP based on ENDF } \\
\text { covariance data }\end{array}$ \\
\hline $\begin{array}{l}\text { Dakota (SNL) } \\
\text { Available as open source }\end{array}$ & $\begin{array}{l}\text { Suite of mathematical and statistical tools that interface to a number of } \\
\text { computational models }\end{array}$ \\
\hline $\begin{array}{l}\text { SCALE/Sampler (ORNL) } \\
\text { Available in SCALE 6.2 }\end{array}$ & $\begin{array}{l}\text { Performs uncertainty analysis for any SCALE calculation. Samples } \\
\text { multigroup cross sections, fission product yields, and decay data based } \\
\text { on extended SCALE covariance libraries. Samples from user input } \\
\text { distributions and correlations for any value in the input file, including } \\
\text { geometry, materials, sources, etc. Provides uncertainty analysis for } \\
\text { criticality, depletion, shielding, activation, decay, and more. }\end{array}$ \\
\hline
\end{tabular}

Sensitivity Analysis Tools

\begin{tabular}{|l|l|}
\hline Tool & Capabilities \\
\hline SCALE/TSUNAMI (ORNL) & $\begin{array}{l}\text { Suite of tools for sensitivity analysis, uncertainty analysis, similarity } \\
\text { assessment for validation, nuclear data and experiment assimilation, } \\
\text { computational bias determination, gap analysis, and more. Includes } \\
\text { continuous-energy and multigroup group sensitivity and uncertainty } \\
\text { analysis for keff and reaction rate calculations for 3D systems using KENO } \\
\text { Monte Carlo code. Includes multigroup group sensitivity and uncertainty } \\
\text { analysis for keff and reaction rate calculations for 1D and 2D systems using } \\
\text { XSDRNPM and NEWT deterministic codes. }\end{array}$ \\
\hline $\begin{array}{l}\text { MCNP/Whisper (LANL) } \\
\text { Available in MCNP 6.2 }\end{array}$ & $\begin{array}{l}\text { Suite of tools for creating bounding estimates of computational biases for } \\
\text { criticality safety assessment based on a combination of statistical and } \\
\text { sensitivity/uncertainty gap analysis. Includes continuous-energy } \\
\text { sensitivity and uncertainty analysis of keff calculations for 3D systems } \\
\text { using MCNP Monte Carlo code. }\end{array}$ \\
\hline $\begin{array}{l}\text { SCALE/ORSEN(ORNL) } \\
\text { Available as beta release on request }\end{array}$ & $\begin{array}{l}\text { Sensitivity analysis version of ORIGEN that provides sensitivity of output } \\
\text { isotopics to input nuclear data values and incorporates SCALE covariance } \\
\text { data for uncertainty quantification. }\end{array}$ \\
\hline
\end{tabular}

\section{A.9.2 Covariance Data}

Covariance data provide a mathematical representation of the uncertainty in nuclear data parameters. The covariance form provides the variance in an individual quantity as well at the shared uncertainty between multiple quantities. For, example, where many reaction cross sections are measured using the same isotopic sample, and where there is an uncertainty in the sample mass or purity, all cross sections obtained from this sample would have a shared uncertainty component. The availability of covariance data for reaction cross sections, neutron multiplicity, and fission energy distributions has been increasing in recent years, with some data now available for approximately one-half of the nuclides in ENDF, a substantial increase from the few dozen nuclides with some form of covariance data as recently as ENDF/B-VII.0. Additionally, collaborative initiatives have generated low-fidelity covariance data to provide some information to fill in many of the gaps in the ENDF library. Some specific projects have generated specialized libraries for other types of nuclear data, such as fission product yields, decay constants, and extended reactions such as $(\alpha, n)$, but these data are not yet included in the ENDF or ENSDF evaluations. 
Data for other processes such as angular distributions, spontaneous fission, gamma emissions, and more, are not available.

Through testing of the available high-fidelity covariance data distributed with ENDF, it has been recognized that the covariance data may exaggerate the uncertainty in the computed results. When propagated to an uncertainty in the $k_{\text {eff }}$ value in hundreds of criticality safety benchmark experiments, the uncertainty due to covariance data are often a factor of two or three greater than the standard deviation of the distribution of results. Through extended discussions within CSEWG and other groups, it has been acknowledged that data evaluators often generate covariance data using the distribution of observables from differential measurements. In processing the mean values for the cross section, choices are made that improve the performance of the library for selected criticality benchmarks of interest through an expert-based, not mathematically based, calibration process. The results are a library with mean values that perform well for the selected benchmarks and covariance data that represent the distribution of the differential measurement prior to calibration. The ENDF/B-VIII.0 library now includes a disclaimer:

"Comments about the covariance in current ENDF evaluations.

1. The covariance data in the ENDF evaluations represents uncertainties and correlations in differential data.

2. The use of this covariance to calculate uncertainties for integral quantities such as $k_{\text {eff }}$ will usually result in an overestimate of the uncertainty.

3. The recommended methodology to overcome this problem is to adjust the covariance to add information from set of integral data that represents the physics of the system for which the adjusted covariance will be used.

4. More information on this topic: https://www.oecd-nea.org/science/wpec/sg33/

5. CSEWG is currently studying the best covariance representation for future releases.”

\section{A.9.3 Plan of Action}

The overwhelming response from the community is that the ability to propagate uncertainties for all data to all calculated results would be a game changer in the use of analytical methods for applications of interest, including correlated detectors, decay chain studies, quantification of special nuclear materials, and characterization of reactor burnup. These tools must be robust and easy for analysts to use. Sustaining user support and training must also be available. Before this vision can be realized, many steps must be taken.

1. Review of quality and use of existing covariance data

a. Covariance data generally represent uncertainty in differential measurement, not in cross section mean value provided in evaluation.

b. ENDF/B-VIII.0 recommends the use of an application-specific adjusted covariance library, so the procedure and analysis workflow must be established.

2. Creation and testing of additional covariance data prioritized by application / sensitivity

a. Prompt data

b. Multiplicity data

c. Neutron cross sections

d. Prompt fission data

e. Delayed data

f. Spontaneous fission

g. Gamma emission

h. Decay data

i. Fission yields

j. Emission $(\alpha, n)$ 
3. Make sensitivity analysis for sensitivity for every tally, every response
a. Eigenvalue
b. Fixed source
c. Reaction rate
d. Transmutation
e. Coincidence / detector responses
f. Use sensitivity to prioritize data and uncertainty needs
g. Use sensitivity to quantify applicability of available benchmarks
h. Enhance and integrate methods to apply data adjustment

4. Support and enhancement of sampling methods
a. Kiwi
b. Sampler
c. Dakota

5. Extend and integrate sampling methods for all data sources
a. Prompt Data
i. Multiplicity data
ii. Neutron cross sections
iii. Prompt fission data
b. Delayed Data
i. Spontaneous fission
ii. Gamma emission
iii. Decay data
iv. Fission yields
v. Emission $(\alpha, n)$

6. Quantify correlation between application cases with shared uncertainties

7. Implement principle component analysis to quantify uncertainties with largest impact to feedback to data needs

\section{A.10 Data Processing and Transport Code Needs}

\section{Session Leads: Bradley T. Rearden, ORNL, and Teresa Bailey, LLNL}

The goal of this discussion was to address supporting work to ensure that the codes can implement new and existing nuclear data in a useful way. High fidelity models require accurate parameter inputs, and low fidelity or missing nuclear data can drive the model uncertainties. However, there are cases where the nuclear data are available, but they are not processed and used properly by the model. The session participants were asked to identify areas where code development was required to support the use of nuclear data for nonproliferation systems. The scope of the discussion included nuclear data evaluation codes, data processing and testing codes, and the multipurpose modeling and simulation codes.

Recent advances in radiation transport and transmutation capabilities and the underlying nuclear data provide new insights into physical processes that can lead to improved approaches for non-proliferation. Increasingly predictive modeling and simulation capabilities provide information such as coincident particle emissions from individual reactions that enable advancements in material characterization. As the usefulness of these calculations is identified by those creating new detection methods, it is necessary to enhance the codes to more broadly provide capabilities to simulate these specialized processes. Sensitivity methods must be enabled in these codes to distinguish reactions and emissions that are important to the physical observable from those that have little impact on the objective. There is also an increasing demand for high-fidelity nuclear data that include associated uncertainties. 


\section{A.10.1 Nuclear Data Processing Codes}

The transformation of the generic ENDF and ENSDF representations of physical phenomena to appropriate data formats for use in radiation transport, transmutation, and uncertainty calculations is known as nuclear data processing. Processing data to a specific format for use in calculations is distinct from nuclear data evaluation, which is applied to generate ENDFs and ENDSFs. Nuclear data are processed in several steps: consolidating data from different physical representations, including thermal scattering law, resolved resonances, unresolved resonances, particle emissions, and more. The processing codes also provide temperature corrections to the fundamental data through Doppler broadening. For multigroup (MG) data, the processing codes allow the user to enter an energy bin structure and a flux weighting function. These are applied as the continuous-energy (CE) data are averaged into the requested group structure.

An important advancement for the ENDF community is the transformation from the current limiting capabilities of the ENDF-6 format for storing nuclear data to the more dynamic General Nuclear Data Structure (GNDS) format. In addition to these widely used formats, LLNL uses their own internal evaluated nuclear data library (ENDL) format.

\section{A.10.1.1 Transport code library processing}

Processing of the reaction cross sections and fission multiplicity data from ENDF data to multigroup (MG) and continuous energy (CE) formats needed for transport codes is performed with tools from LANL, LLNL, and ORNL, as shown below in Table A.10-1.

Table A.10-1. ENDF Reaction Cross Section and Fission Multiplicity Processing Tools

\begin{tabular}{ll}
\hline Tool & Capabilities \\
\hline NJOY (LANL) & Creates MG and CE neutron and gamma ACE libraries for MCNP \\
& from ENDF-6 formatted data \\
FUDGE (LLNL) & Creates MG and CE neutron and gamma libraries for COG, \\
& Mercury, and Ardra from ENDF, ENDL and GNDS formatted data \\
AMPX (ORNL) & Creates MG and CE neutron and gamma libraries for SCALE from \\
& ENDF-6 and GND formatted data \\
\hline
\end{tabular}

\section{A.10.1.2 Transmutation data processing}

The generation of nuclear data libraries for activation, depletion, and decay requires many steps in combining data from many sources. While ENDF provides reaction data for approximately 400 nuclides, the Joint Evaluated Fission and Fusion File (JEFF) from the Organisation for Economic Cooperation and Development (OECD) Nuclear Energy Agency (NEA) provides activation data for over 2,000 nuclides. The generation of transmutation data for a depletion code like ORIGEN requires the following data:

- Decay data

o ENDF

O Natural isotopic abundances (NIST database)

o ICRP 72 inhalation dose coefficients, EPA Report 12, external exposure

- Neutron reaction cross section data

o JEFF special purpose activation file

o ENDF

- Fission product yields: ENDF

- Photon emission line-energy data 


\section{O ENSDF}

O ENDF

- Neutron emission libraries

o SOURCES 4C code

o Spontaneous fission prompt and delayed neutron emission multiplicity and energy spectrum

$0 \quad(\alpha, n)$ cross sections and stopping powers, neutron emission spectrum

Currently, AMPX is the only data processing code with the capability to create transmutation libraries. AMPX combines data from many sources to create transition matrix, fission product yield, decay, and emission files.

\section{A.10.1.3 Nuclear data uncertainty processing}

The processing of uncertainties in nuclear data to covariance matrices useable in sensitivity/uncertainty analysis is only available in limited forms using NJOY and AMPX. Work is needed to increase the amount of data in which covariances are available and to improve the capabilities of processing codes to create formats usable by the neutron transport codes.

\section{A.10.2 Transmutation codes}

The MCNP can couple with CINDER for depletion, and SCALE can couple with ORIGEN for activation, depletion, and decay calculations. The sensitivity and uncertainty capabilities of ORSEN are being developed within ORIGEN to provide the sensitivity of output quantities such as fission products and emission spectra due to uncertainties in the nuclear data used in the calculation.

\section{A.10.3 Code development needs}

The need for improved detector response models is not a nuclear data topic, but it received significant attention during the session. Many detector models require event-by-event charged particle transport, which is computationally costly and difficult to implement. There was discussion of linking transport codes with detector response codes such as GADRAS or to physics codes such as GEANT. This is a large undertaking, but the need is important, so it should be addressed in a separate venue.

Recently, the fission physics codes FREYA and CGMF have been incorporated into MCNP, and their ability to model prompt neutron and gamma emissions from fission was improved. Once these models are validated, they can be used to model benchmark systems and the results used to test and validate new fission data. Future work is needed to provide the timing information for correlated neutrons and gammas to enable models of correlated particle detection systems. The timing of fission chains is also considered important.

Modeling of delayed gamma and neutron emissions was discussed, and codes such as ORIGEN can provide the decay data to the neutron transport model, including capture gamma emission. There was a consensus among the group that there are a lot of inconsistencies between ENDF and ENSDF databases; providing better integration of the two databases would help many applications.

Multiple improvements would be useful for a variety of applications. Prioritizing these improvements for the codes maintained at LANL, ORNL and LLNL may not be possible. The prioritization should come from the specific application supported by the code. The best mechanism to ensure that nuclear data are available to the user is to include the required code development in parallel to the nuclear data improvements. In some cases, the nuclear data are already available in the database but not fully implemented by the transport codes. 
Each proposed nuclear data improvement should examine the following infrastructure requirements:

1. Theoretical models and evaluation codes to evaluate the data

2. A mechanism to provide covariance data

3. Nuclear data processing capabilities for new types of data sets

4. Transport code development to ensure the data is usable

5. Validation of new physics models within the codes to ensure the data is used correctly

6. Data testing

\section{A.11 Targets, Facilities and Detector System}

\section{Session Leads: Jason Burke, LLNL, and Todd Bredeweg, LANL}

The primary goal of this session was to identify nuclear data target needs for future experiments. In addition, general nuclear data capabilities in the form of facilities and detection systems were discussed. The participants' input on target needs and capabilities will be covered in this summary. The existing facilities were previously documented in the Nuclear Data Needs and Capabilities for Applications workshop and are described here: http://bang.berkeley.edu/events/ndnca/whitepaper/ but are not documented in this summary; however, some specific needs will be identified.

The acquisition of isotopically pure stable and radioactive isotopes for nuclear data experiments and research in general is expensive, and the supply is limited. The US Isotopes Program provides most of the targets, but supply of many isotopes is limited because the US dismantled its enrichment and radioisotope target production capabilities in the 1980s. A new enrichment capability for stable isotopes at ORNL is now operational and is a positive step towards providing the community the most important stable isotopes, but radioisotope enrichment and target production are still lacking.

\section{A.11.1 Stable isotope targets}

\section{A.11.1.1 Enriched stable isotope availability}

Until recently, many stable isotope targets could be leased for an experiment and returned to the isotope program. Unfortunately, new restrictions have been placed on leased targets, limiting the amount of activation created in the target. This appears to be a reasonable policy to preserve the stock of materials, but it creates a cost burden on programs funding nuclear data because the purchase price for stable isotopes is about ten times the lease price. The workshop participants recommend that the policy be revisited to ensure that important experiments are not impacted.

\section{A.11.1.2 Target fabrication of stable isotopes}

There are currently extensive target fabrication capabilities at ORNL and ANL. Modern capabilities should be investigated that may be able to be transferred to radioisotope target production.

\section{A.11.2 Radioisotope targets}

\section{A.11.2.1 Enriched radioisotope availability}

Many enriched radioisotopes are typically not available unless they are relatively long-lived and are part of existing stock, or unless they can be produced and enriched by chemical separation, as when a decay product is milked from a radioactive material. Recently, LANL and ORNL have examined the requirements to create a capability to enrich milligram to gram quantities of radioisotopes to support 
research needs. INL has the capability to separate microgram quantities of radioisotopes, but this does not meet most requirements. In addition, the capability to harvest small amounts of radioisotopes at FRIB is being investigated.

\section{A.11.2.2 Target fabrication of radioisotopes}

Electrodeposition is currently the go-to method for radioisotope target fabrication. However, it is not ideal for many applications. For example, it does not provide a pure, thin target for fission yield experiments because the fission fragments lose too much energy within the target material. In this case, vacuum evaporation is the ideal method, but there is no vacuum evaporation capability to support target production requests through the isotope program. Participants consider it important to reestablish the capability to support nuclear data experiments. In addition, modern methods for thin film production should be examined.

Metal foil targets require small-scale metal conversion of pure isotopes, along with hot rolling and welding between aluminum foils. There is limited capability at LANL and LLNL, and enhancing this capability should also be supported.

\section{A.11.2.3 Target characterization}

Once a target is produced, it must be carefully characterized to determine the mass, thickness, and evenness of deposition. This step is very important to reduce experimental uncertainties. This may require dedicated equipment to avoid contamination from radioisotope targets.

\section{A.11.3 Recommendations}

- Provide researchers with access to a list of available materials from the isotope catalog. Providing this information on a need-to-know basis can allow researchers and experimenters to more effectively plan research programs.

- Create a shareable database of samples and isotope enrichments at each of the labs to enable target sharing.

- Provide isotopically pure $\mathrm{U}, \mathrm{Pu}$, and minor actinides in microgram to gram quantities. o Participation in the new CIELO international collaboration will require targets of ${ }^{238-242} \mathrm{Pu}$

- Create a searchable tool documenting available fluxes and energies at various facilities similar to or in collaboration with the Research and Test Facilities Database (RTFDB) maintained by NEA. o Include a statement of importance/relevance of the accelerator facilities and reactors.

o Include neutron spectra and fluxes from reactors/neutron sources. (Much of this information is documented in the NDNCA white paper at: http://bang.berkeley.edu/events/ndnca/whitepaper/)

Needs for new facilities or facility upgrades were discussed, and those noted by the participants include:

- A dedicated facility for neutron scattering with angular distributions

- A place to produce and measure short-lived actinides in the same location, because shipping a short-lived target is not feasible.

- Installation of a rabbit at the DAF for Godiva/Flattop to enable gamma measurements of fission yields at short time scales. 
APPENDIX B. MEETING AGENDA AND PRESENTATIONS 



\section{B.1 NDREW AGENDA}

Tuesday, January 23, $2018 \cdot 1608$ Rhode Island Avenue NW • Washington, DC • Rooms A, B \& C

\begin{tabular}{|c|c|c|}
\hline 0745 & $\begin{array}{l}\text { Registration \& Continental Breakfast (included in meal } \\
\text { fee) }\end{array}$ & \\
\hline 0830 & NNSA Welcome \& Opening Remarks & $\begin{array}{r}\text { Craig Sloan, Office of Proliferation } \\
\text { Detection, DNN R\&D, NNSA }\end{array}$ \\
\hline 0845 & Historical Context, Meeting Structure and Goals & Catherine Romano, ORNL \\
\hline 0910 & $\begin{array}{l}\text { DOE-SC-NP Interagency Coordination and the } \\
\text { Upcoming FOA }\end{array}$ & $\begin{array}{r}\text { Tim Hallman, Associate Director of } \\
\text { Science for Nuclear Physics }\end{array}$ \\
\hline 0925 & Interagency Coordination: DTRA, DHS-DNDO, AFTAC & Interagency Program Managers \\
\hline 1005 & Break & All \\
\hline 1025 & International Coordination: IAEA & $\begin{array}{l}\text { Arjan Koning, Section Head, } \\
\text { Nuclear Data Section, IAEA }\end{array}$ \\
\hline 1040 & $\begin{array}{l}\text { NA-22 Program Management Perspectives and Panel } \\
\text { Introduction }\end{array}$ & $\begin{array}{r}\text { Donald Hornback, Office of } \\
\text { Proliferation Detection, DNN R\&D, } \\
\text { NNSA }\end{array}$ \\
\hline 1055 & $\begin{array}{l}\text { Panel } \\
\text { - Impact of Nuclear Data on Nonproliferation Missions: } \\
\text { User Perspectives } \\
\text { - Use of Uncertainty Quantification to Identify Nuclear } \\
\text { Data Needs } \\
\text { - The Nuclear Data Pipeline } \\
\text { - Audience Q\&A }\end{array}$ & $\begin{array}{r}\text { Teresa Bailey, LLNL } \\
\text { Jerome Verbeke, LLNL } \\
\text { Lori Metz, PNNL } \\
\text { Brad Rearden, ORNL } \\
\text { David Brown, BNL }\end{array}$ \\
\hline 1215 & Lunch (included in meal fee) \& Group Photo & All \\
\hline 1330 & $\begin{array}{l}\text { Group Roadmapping Session } 1 \\
\text { - Topic 1A: Uncertainty, Sensitivity, \& Covariance (Rms } \\
\text { A\&B) } \\
\text { - Topic 1B: Neutron Capture and Associated Spectra } \\
\text { (Rm C) } \\
\text { - Topic 1C: Fission I, Independent \& Cumulative Yields } \\
\text { (Aud) }\end{array}$ & $\begin{array}{r}\text { 1A Session Leader: Brad Rearden, } \\
\text { ORNL } \\
\text { 1B Session Leader: Lee Bernstein, } \\
\text { LBNL } \\
\text { 1C Session Leader: Patrick Talou, } \\
\text { LANL }\end{array}$ \\
\hline 1455 & Break & All \\
\hline 1515 & Group Roadmapping Session 1 (continued) & \\
\hline
\end{tabular}


Wednesday, January 24, $2018 \cdot 1608$ Rhode Island Avenue NW • Washington, DC • Rooms A, B \& C

\begin{tabular}{|c|c|c|}
\hline 0745 & $\begin{array}{l}\text { Registration (if you didn't attend Day 1) \& Continental } \\
\text { Breakfast (included in meal fee) }\end{array}$ & \\
\hline 0830 & $\begin{array}{ll} & \text { Group Roadmapping Session } 2 \\
\text { - } & \text { Topic 2A: Gamma-Induced Reactions (Rm C) } \\
\text { - } & \text { Topic 2B: Inelastic Neutron Scattering \& Associated Spectra } \\
& \text { (Rms A\&B) } \\
\text { - } & \text { Topic 2C: Fission II, Prompt Gammas \& Neutrons } \\
& \text { (Auditorium) }\end{array}$ & $\begin{array}{r}\text { 2A Session Leader: Brian Quiter, } \\
\text { LBNL } \\
\text { 2B Session Leader: Lee } \\
\text { Bernstein, LBNL and UC } \\
\text { Berkeley } \\
\text { 2C Session Leader: Sara Pozzi, } \\
\text { University of Michigan }\end{array}$ \\
\hline 1000 & Break & All \\
\hline 1020 & Group Roadmapping Session 2 (continued) & \\
\hline 1200 & Lunch (included in meal fee) & All \\
\hline 1315 & $\begin{array}{l}\text { Group Roadmapping Session } 3 \\
\text { - Topic 3A: (a,n) Reactions (Rm C) } \\
\text { - Topic 3B: Targets, Facilities and Detector System } \\
\text { (Auditorium) } \\
\text { - Topic 3C: Fission III, Decay Data (Rms A\&B) }\end{array}$ & $\begin{array}{r}\text { 3A Session Leader: Matt Devlin, } \\
\text { LANL } \\
\text { 3B Session Leaders: Jason } \\
\text { Burke, LLNL } \\
\text { \& Todd Bredeweg, LANL } \\
\text { 3C Session Leader: } \\
\text { Libby McCutchan, BNL }\end{array}$ \\
\hline 1455 & Break & All \\
\hline 1515 & Group Roadmapping Session 3 (continued) & \\
\hline 1700 & Adjourn Day 2 & \\
\hline 1715 & Optional No Host Social & $\begin{array}{r}\text { Beacon Hotel Grilll } \\
1615 \text { Rhode Island Avenue } \\
\text { (202) 872-1126 / } \\
\text { www.beaconhotelwdc.com }\end{array}$ \\
\hline
\end{tabular}


Thursday, January 25, $2018 \cdot 1608$ Rhode Island Avenue NW • Washington, DC • Rooms A, B \& C

\begin{tabular}{|c|c|c|}
\hline 0745 & $\begin{array}{l}\text { Registration (if you didn't attend Day } 1 \text { or 2) \& Continental } \\
\text { Breakfast (included in meal fee) }\end{array}$ & \\
\hline 0830 & $\begin{array}{l}\text { Group Roadmapping Session } 4 \\
\text { - } \quad \text { Topic 4A: Development of Benchmark Exercises (Rm C) } \\
\text { - } \quad \text { Topic 4B: Data Processing \& Transport Code Needs (Rms } \\
\text { A\&B) } \\
\text { - } \quad \text { Topic 4C: Actinide Cross Sections (Auditorium) }\end{array}$ & $\begin{array}{r}\text { 4A Session Leaders: Sean } \\
\text { Stave, NA-24 } \\
\text { \& Rian } \\
\text { Bahran, LANL } \\
\text { 4B Session Leaders: Brad } \\
\text { Rearden, ORNL \& Teresa } \\
\text { Bailey, LLNL } \\
\text { 4C Session Leaders: Susan } \\
\text { Hogle, ORNL } \\
\text { \& Lee Bernstein, LBNL }\end{array}$ \\
\hline 1000 & Break & All \\
\hline 1020 & Group Roadmapping Session 4 (continued) & \\
\hline 1200 & Lunch (included in meal fee) & All \\
\hline 1300 & $\begin{array}{l}\text { Presentation of Key Outcomes from Group Sessions to } \\
\text { Program Managers }\end{array}$ & Session Leaders \\
\hline 1515 & Break & All \\
\hline 1535 & Q\&A Session & $\begin{array}{r}\text { Facilitated by Catherine } \\
\text { Romano, ORNL }\end{array}$ \\
\hline 1650 & Workshop Closing and Next Steps & Catherine Romano, ORNL \\
\hline 1700 & Adjourn Final Day 3 & \\
\hline
\end{tabular}





\section{B.2 ATTENDEES}

\begin{tabular}{|c|c|c|}
\hline Last & First & Affiliation \\
\hline Ashenfelter & Timothy & DHS/CWMDT/DNDO \\
\hline Ault & Timothy & NNSA, Defense Nuclear Nonproliferation R\&D \\
\hline Bahran & Rian & Los Alamos National Laboratory \\
\hline Bailey & Teresa & Lawrence Livermore National Laboratory \\
\hline Barnes & Frank & Dept of Energy, Office of Nuclear Physics \\
\hline Beck & Bret & Lawrence Livermore National Laboratory \\
\hline Bernstein & Lee & Lawrence Berkeley National Lab/UC Berkeley \\
\hline Berrington & Matthew & Ministry of Defense \\
\hline Blackston & Matthew & Oak Ridge National Laboratory \\
\hline Blain & Ezekiel & Rensselaer Polytechnic Institute \\
\hline Bowen & Douglas & Oak Ridge National Laboratory \\
\hline Bredeweg & Todd & Los Alamos National Laboratory \\
\hline Brown & David & Brookhaven National Laboratory \\
\hline Bulgac & Aurel & University of Washington \\
\hline Burke & Jason & Lawrence Livermore National Laboratory \\
\hline Champagne & Arthur & Triangle Universities Nuclear Laboratory \\
\hline Chapman & Chris & Oak Ridge National Laboratory \\
\hline Conlin & Jeremy & Los Alamos National Laboratory \\
\hline Couture & Aaron & Los Alamos National Laboratory \\
\hline Croft & Stephen & Oak Ridge National Laboratory \\
\hline Cverna & Frank & Nevada National Security Site \\
\hline Danon & Yaron & Rensselaer Polytechnic Institute \\
\hline Devlin & Matthew & Los Alamos National Laboratory \\
\hline Escher & Jutta & Lawrence Livermore National Laboratory \\
\hline Eskin & Leo & StarCore Nuclear \\
\hline Favalli & Andrea & Los Alamos National Laboratory \\
\hline Fei & Tingzhou & Argonne National Laboratory \\
\hline Fisher & Brian & $\begin{array}{l}\text { The Johns Hopkins University Applied Physics } \\
\text { Laboratory }\end{array}$ \\
\hline Flaska & Marek & Pennsylvania State University \\
\hline Friedrich & Stephan & Lawrence Livermore National Laboratory \\
\hline Funk & Dan & $\begin{array}{l}\text { US Department of Energy, Office of Nuclear } \\
\text { Energy }\end{array}$ \\
\hline Glaser & Joseph & US Department of Energy,Isotope Program \\
\hline
\end{tabular}




\begin{tabular}{|c|c|c|}
\hline Last & First & Affiliation \\
\hline Goldhagen & Paul & $\begin{array}{l}\text { DHS National Urban Security Technology } \\
\text { Laboratory }\end{array}$ \\
\hline Grimes & Steven & Ohio University \\
\hline Guber & Klaus & Oak Ridge National Laboratory \\
\hline Hallman & Tim & $\begin{array}{l}\text { US Department of Energy, Office of Science, } \\
\text { Nuclear Physics }\end{array}$ \\
\hline Hawari & Ayman & North Carolina State University \\
\hline Heffner & Mike & Lawrence Livermore National Laboratory \\
\hline Hogle & Susan & Oak Ridge National Laboratory \\
\hline Horak & William & Brookhaven National Laboratory \\
\hline Hornback & Donald & NNSA, Defense Nuclear Nonproliferation R\&D \\
\hline Hurst & Aaron & University of California, Berkeley \\
\hline Ingraham & Joanna & Defense Threat Reduction Agency \\
\hline Jackman & Kevin & NNSA, Defense Nuclear Nonproliferation R\&D \\
\hline Jaffke & Patrick & Los Alamos National Laboratory \\
\hline Johnson & Jeffrey & Oak Ridge National Laboratory \\
\hline Kiess & Thomas & NNSA, Defense Nuclear Nonproliferation R\&D \\
\hline Kiff & Scott & Sandia National Laboratories \\
\hline Koning & Arjan & International Atomic Energy Agency \\
\hline Laviolette & Randall & Office of Science, Dept. of Energy \\
\hline Lee & Hye Young & Los Alamos National Laboratory \\
\hline Little & Robert & Los Alamos National Laboratory \\
\hline Mackney & Dan & US Air Force \\
\hline Massey & Thomas & Ohio University \\
\hline Matters & David & Defense Threat Reduction Agency \\
\hline Mattingly & John & North Carolina State University \\
\hline Mattoon & Caleb & Lawrence Livermore National Laboratory \\
\hline McAninch & Jeffrey & Defense Threat Reduction Agency \\
\hline Meierbachtol & Krista & Los Alamos National Laboratory \\
\hline Metz & Lori & Pacific Northwest National Laboratory \\
\hline Milbrath & Brian & Pacific Northwest National Laboratory \\
\hline Miller & Ben & National Nuclear Security Administration \\
\hline Moon & Namdoo & Federal Government \\
\hline Mueller & Kevin & Defense Threat Reduction Agency \\
\hline Mulder & Eben & X-energy \\
\hline Nakae & Leslie & Lawrence Livermore National Laboratory \\
\hline
\end{tabular}




\begin{tabular}{|c|c|c|}
\hline Last & First & Affiliation \\
\hline Nelson & Noel & NNSA, Defense Nuclear Nonproliferation R\&D \\
\hline Nicholson & Andrew & Oak Ridge National Laboratory \\
\hline Nortier & Francois & Los Alamos National Laboratory \\
\hline Ormand & William & Lawrence Livermore National Laboratory \\
\hline Pao & Karen & Defense Threat Reduction Agency \\
\hline Parks & Cecil & Oak Ridge National Laboratory \\
\hline Percher & Catherine & Lawrence Livermore National Laboratory \\
\hline Pereira & Candido & Argonne National Laboratory \\
\hline Petersen & David & Defense Threat Reduction Agency \\
\hline Pickett & Chris & NNSA, Defense Nuclear Nonproliferation R\&D \\
\hline Pigni & Marco & Oak Ridge National Laboratory \\
\hline Pitcher & Eric & Los Alamos National Laboratory \\
\hline Pitts & Neil & UK Ministry of Defence \\
\hline Pozzi & Sara & University of Michigan \\
\hline Quiter & Brian & Lawrence Berkeley National Laboratory \\
\hline Ramos & Christopher & National Nuclear Security Administration \\
\hline Raziano & Donna & Lawrence Livermore National Laboratory \\
\hline Rearden & Bradley & Oak Ridge National Laboratory \\
\hline Redmond & Everett & Nuclear Energy Institute \\
\hline Rhodes & William & National Nuclear Security Administration \\
\hline $\begin{array}{l}\text { Ricard- } \\
\text { McCutchan }\end{array}$ & Elizabeth & Brookhaven National Laboratory \\
\hline Roberts & Roger & Defense Threat Reduction Agency \\
\hline Rolfes & Jeffrey & Defense Threat Reduction Agency \\
\hline Romano & Catherine & Oak Ridge National Laboratory \\
\hline Rundberg & Robert & Los Alamos National Laboratory \\
\hline Schaaff & Thomas & National Nuclear Security Administration \\
\hline Schafer & Jenifer & Colorado School of Mines \\
\hline Schatz & Hendrik & Michigan State University \\
\hline Schillebeeckx & Peter & European Commission, Joint Research Centre \\
\hline Schunck & Nicolas & Lawrence Livermore National Laboratory \\
\hline Scielzo & Nicholas & Lawrence Livermore National Laboratory \\
\hline Shipbaugh & Calvin & Defense Threat Reduction Agency \\
\hline Skutnik & Steve & University of Tennessee-Knoxville \\
\hline Sloan & Craig & NNSA, Defense Nuclear Nonproliferation R\&D \\
\hline
\end{tabular}




\begin{tabular}{|c|c|c|}
\hline Last & First & Affiliation \\
\hline Smalley & Duane & Mission Support and Test Services \\
\hline Smith & Michael & Oak Ridge National Laboratory \\
\hline Snyder & Lucas & Lawrence Livermore National Laboratory \\
\hline Sonzogni & Alejandro & National Nuclear Data Center \\
\hline Stave & Sean & NNSA/NA-241 \\
\hline Talou & Patrick & Los Alamos National Laboratory \\
\hline Thompson & Nicholas & Los Alamos National Laboratory \\
\hline Thompson & Ian & Livermore Laboratory \\
\hline Thompson & Scott & Idaho National Laboratory \\
\hline Tobin & Kenneth & Oak Ridge National Laboratory \\
\hline Tonchev & Anton & Lawrence Livermore National Laboratory \\
\hline Tovesson & Fredrik & NNSA, Defense Nuclear Nonproliferation R\&D \\
\hline Travia & Nicholas & Lawrence Livermore National Laboratory \\
\hline Tremaine & Aaron & SLAC National Accelerator Laboratory \\
\hline Uhlhorn & Susan & Lawrence Livermore National Laboratory \\
\hline Valentine & John & Lawrence Berkeley National Laboratory \\
\hline VanDevender & Brent & Pacific Northwest National Laboratory \\
\hline Vanhoy & Jeffrey & United States Naval Academy \\
\hline Verbeke & Jerome & Lawrence Livermore National Laboratory \\
\hline Vogt & Ramona & Lawrence Livermore National Laboratory \\
\hline Walker & Scottie & NNSA /NA-82 \\
\hline Warren & Glen & Pacific Northwest National Laboratory \\
\hline Weidman & Randall & National Nuclear Security Administration \\
\hline Weltz & Adam & $\begin{array}{l}\text { Defense Threat Reduction Agency, Pennsylvania } \\
\text { State University }\end{array}$ \\
\hline White & Morgan & Los Alamos National Laboratory \\
\hline Wieselquist & William & Oak Ridge National Laboratory \\
\hline $\mathrm{Wu}$ & Ching-Yen & Lawrence Livermore National Laboratory \\
\hline Zerkle & Michael & Naval Nuclear Laboratory \\
\hline Noonan & Bill & $\begin{array}{l}\text { John Hopkins University, Applied Physics } \\
\text { Laboratory }\end{array}$ \\
\hline Hunt & Alan & $\begin{array}{l}\text { John Hopkins University, Applied Physics } \\
\text { Laboratory }\end{array}$ \\
\hline
\end{tabular}

